DFTT $1 / 2000$

IFIC/00-12, FTUV/00-11

LAPTH $-779 / 2000$

\title{
Further investigation of a relic neutralino as a possible origin of an annual-modulation effect in WIMP direct search
}

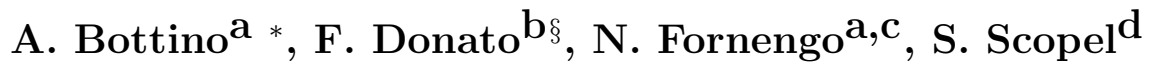 \\ a Dipartimento di Fisica Teorica, Università di Torino \\ and INFN, Sez. di Torino, Via P. Giuria 1, I-10125 Torino, Italy \\ ${ }^{b}$ Laboratoire de Physique Théorique LAPTH, B.P. 110, F-74941 \\ Annecy-le-Vieux Cedex, France \\ ${ }^{c}$ Instituto de Física Corpuscular - C.S.I.C. \\ Departamento de Física Teòrica, Universitat de València \\ Edificio Institutos de Paterna, Apt 2085, 46071 Valencia, Spain \\ $d$ Instituto de Física Nuclear y Altas Energías, Facultad de Ciencias, \\ Universidad de Zaragoza, Plaza de San Francisco s/n, E-50009 Zaragoza, Spain
}

\begin{abstract}
We analyze the annual-modulation effect, measured by the DAMA Collaboration with the new implementation of a further two-years running, in the context of a possible interpretation in terms of relic neutralinos. We impose over the set of supersymmetric configurations, selected by the annualmodulation data, the constraints derived from WIMP indirect measurements, and discuss the features of the ensuing relic neutralinos. We critically discuss the sources of the main theoretical uncertainties in the analysis of event rates for direct and indirect WIMP searches.
\end{abstract}

Typeset using REVTEX

*E-mail: bottino@to.infn.it, donato@lapp.in2p3.fr, fornengo@flamenco.ific.uv.es, scopel@posta.unizar.es

${ }^{\S}$ INFN Post-doctoral Fellow 


\section{INTRODUCTION}

The effect of annual modulation measured by the DAMA Collaboration in its WIMP direct search experiment with a $\mathrm{NaI}(\mathrm{Tl})$ detector and reported in Ref. [1] was analyzed in terms of relic neutralinos in Refs. [2 [4]. In these papers, we proved that this interpretation is compatible with the DAMA data, and entails a relic neutralino which might have the role of a major component of dark matter in the Universe, especially when the uncertainties affecting the evaluation of the neutralino-nucleon cross section are taken into account (i] ). We have also presented in detail other physical properties of such a neutralino, both in a Minimal Supersymmetric extension of the Standard Model [2 [- 1$]$ and in supergravity schemes [2], and we have outlined how indirect measurements of WIMPS (low-energy antiprotons in cosmic rays and up-going muon fluxes from the center of the Earth and from the Sun) may bring further information [2], by way of constraints on the supersymmetric configurations derived from the DAMA annual-modulation results [5].

New data, collected by the DAMA Collaboration in a further two-year running of the $\mathrm{NaI}(\mathrm{Tl})$ experiment for an exposure of $38475 \mathrm{~kg} \cdot$ day, and now presented in Ref. [6], confirm their previous finding of an annual-modulation effect, which does not appear to be related to any possible source of random systematics. Taking together all (old and new) samples of data for a total exposure of $57986 \mathrm{~kg}$ - day, the effect turns out to be at a $4 \sigma$ C.L. Performing a maximum likelihood analysis in terms of $m_{\chi}$ and $\xi \sigma_{\text {scalar }}^{\text {(nuclen) }}$, where $m_{\chi}$ is the WIMP mass, $\sigma_{\text {scalar }}^{\text {(nucleon }}$ is the WIMP-nucleon scalar elastic cross-section, and $\xi=\rho_{\chi} / \rho_{l}$ is the WIMP fractional amount of local non-baryonic dark-matter density $\rho_{l}$, the DAMA Collaboration presents a $3 \sigma$ C.L. annual-modulation region, in the plane $m_{\chi}-\xi \sigma_{\text {scalar }}^{\text {(nuclon) }}$, whose actual size depends on whether or not the upper-bound constraints previously obtained by the same Collaboration [7] are included, and on the values assigned to the galactic astrophysical velocities. For the purpose of the analysis carried out in the present paper, among the regions presented in Ref. [6] we select the one, which is obtained from the annual-modulation data, by including the upper-bound constraints of Ref. [7], by setting $\rho_{l}$ at the standard reference value: $\rho_{l}=0.3 \mathrm{GeV} \mathrm{cm}{ }^{-3}$, and by taking into account uncertainties in the astrophysical velocities of the usual galactic Maxwellian distribution $\left(170 \mathrm{~km} \mathrm{~s}^{-1} \leq v_{0} \leq 270 \mathrm{~km} \mathrm{~s}^{-1} ; v_{\text {esc }}\right.$ $=450-650 \mathrm{~km} \mathrm{~s}^{-1}$; where $v_{0}$ is the rotational velocity of the local system at the position of the solar system and $v_{\text {esc }}$ is the galactic escape velocity). This region is the one shown in Fig. 1 (should one include also a bulk rotation of the dark matter halo [8,3], this region would elongate along the horizontal axis up to $m_{\chi} \sim 230 \mathrm{GeV}$ [6]). In this figure we also show the contour lines for the three values $v_{0}=170,220,270 \mathrm{~km} \mathrm{~s}^{-1}$, separately [9]. In the comparison of the experimental data with the theoretical evaluations one has to further consider the uncertainty in $\rho_{l}: 0.1 \mathrm{GeV} \mathrm{cm}^{-3} \leq \rho_{l} \leq 0.7 \mathrm{GeV} \mathrm{cm}^{-3}$ [10,[1]. Fig. 2 displays how the DAMA annual-modulation region shifts along the vertical axis, as the value of $\rho_{l}$ is varied within its uncertainty range. The four panels correspond to the representative values: $\rho_{l}=0.1,0.3,0.5,0.7 \mathrm{GeV} \mathrm{cm}^{-3}$. In Fig. 2 , as well as in all subsequent figures, where experimental results of direct and indirect WIMP measurements are compared with theoretical evaluations, separate panels are used for the four representative values of $\rho_{l}$.

In the present paper we investigate the implications of the DAMA data with the total exposure of $57986 \mathrm{~kg}$ · day in terms of relic neutralinos, along the lines previously developed 
in Refs. 22 [4. We single out the set of the supersymmetric configurations compatible with the DAMA data, and then apply to this set, denoted as set $S$, the constraints derived from experimental indirect searches for WIMPs (up-going muons at neutrino telescopes and antiprotons in cosmic rays). In this analysis we incorporate recent, and quite significant, theoretical and experimental developments.

The supersymmetric theoretical framework adopted here is the Minimal Supersymmetric extension of the Standard Model (MSSM) [12], which conveniently describes the supersymmetric phenomenology at the electroweak scale, without too strong theoretical assumptions. This model has been extensively used by a number of authors for evaluations of the neutralino relic abundance and detection rates (a list of references may be found, for instance, in 113 ).

The neutralino is defined as the lowest-mass linear superposition of photino $(\tilde{\gamma})$, zino $(\tilde{Z})$ and the two higgsino states $\left(\tilde{H}_{1}^{\circ}, \tilde{H}_{2}^{\circ}\right): \chi \equiv a_{1} \tilde{\gamma}+a_{2} \tilde{Z}+a_{3} \tilde{H}_{1}^{\circ}+a_{4} \tilde{H}_{2}^{\circ}$.

The MSSM contains three neutral Higgs fields: two of them $(h, H)$ are scalar and one $(A)$ is pseudoscalar. At the tree level the Higgs sector is specified by two independent parameters: the mass of one of the physical Higgs fields, which we choose to be the mass $m_{A}$ of the neutral pseudoscalar boson, and the ratio of the two vacuum expectation values, defined as $\tan \beta \equiv\left\langle H_{2}\right\rangle /\left\langle H_{1}\right\rangle$. Once radiative corrections are introduced, the Higgs sector depends also on the squark masses through loop diagrams. The radiative corrections to the neutral and charged Higgs bosons, employed in the present paper, are taken from Refs. 14]. The other parameters of the model are defined in the superpotential, which contains all the Yukawa interactions and the Higgs-mixing term $\mu H_{1} H_{2}$, and in the soft-breaking Lagrangian, which contains the trilinear and bilinear breaking parameters and the soft gaugino and scalar mass terms.

To cast the MSSM, which originally contains a large number of parameters, into a form adequate for phenomenology, we follow the common procedure of introducing a set of restrictive assumptions at the electroweak scale: a) all trilinear parameters are set to zero except those of the third family, which are unified to a common value $A ; \mathrm{b}$ ) all squarks and sleptons soft-mass parameters are taken as degenerate: $m_{\tilde{l}_{i}}=m_{\tilde{q}_{i}} \equiv m_{0}$, c) the gaugino masses are assumed to unify at $M_{G U T}$, and this implies that the $U(1)$ and $S U(2)$ gaugino masses are related at the electroweak scale by $M_{1}=(5 / 3) \tan ^{2} \theta_{W} M_{2}$.

Once these conditions are implemented in the model, the supersymmetric parameter space consists of six independent parameters. We choose them to be: $M_{2}, \mu, \tan \beta, m_{A}, m_{0}, A$ and vary these parameters in the following ranges: $10 \mathrm{GeV} \leq M_{2} \leq 1 \mathrm{TeV}, 10 \mathrm{GeV} \leq|\mu| \leq$ $1 \mathrm{TeV}, 80 \mathrm{GeV} \leq m_{A} \leq 1 \mathrm{TeV}, 100 \mathrm{GeV} \leq m_{0} \leq 1 \mathrm{TeV},-3 \leq A \leq+3,1 \leq \tan \beta \leq 50$. We remark that the values taken here as upper limits of the ranges for the dimensional parameters, $M_{2}, \mu, m_{0}, m_{A}$, are inspired by the upper bounds which may be derived for these quantities in SUGRA theories, when one requires that the electroweak symmetry breaking, radiatively induced by the soft supersymmetry breaking, does not occur with excessive fine tuning (see Ref. [15] and references quoted therein).

We have further constrained our parameter space, by taking into account all the new experimental limits obtained from accelerators on supersymmetric and Higgs searches (LEP2 [16], CDF [17]). Notice that the new bounds from LEP2 and CDF constrain now rather severely the susy space, especially in the region of interest for direct detection (small $m_{h}$ and, partially, large $\tan \beta$ [17]). 
Moreover, the constraints due to the $b \rightarrow s+\gamma$ process [18] have been taken into account. In our analysis, the inclusive decay rate $\operatorname{BR}\left(B \rightarrow X_{s} \gamma\right)$ is calculated with corrections up to the leading order. Next-to-leading order corrections [19] are included only when they can be applied in a consistent way, i.e. both to standard-model and to susy diagrams. We require that our theoretical evaluation for $\mathrm{BR}\left(B \rightarrow X_{s} \gamma\right)$ is within the range: $1.96 \times 10^{-4} \leq$ $\operatorname{BR}\left(B \rightarrow X_{s} \gamma\right) \leq 4.32 \times 10^{-4}$. This range is obtained by combining the experimental data of Refs. 220.21] at 95\% C.L. and by adding a theoretical uncertainty of $25 \%$, whenever the still incomplete next-to-leading order susy corrections cannot be applied.

Our parameter space has been further constrained by the request that the Lightest Supersymmetric Particle (LSP) is the neutralino, rather than the gluino or squarks or sleptons. The current upper bound for cold dark matter may be establish as $\Omega_{C D M} h^{2} \lesssim 0.3$ ( $h$ is the usual Hubble parameter, defined in terms of the present-day value $H_{0}$ of the Hubble constant as $\left.h \equiv H_{0} /\left(100 \mathrm{~km} \mathrm{~s}^{-1} \mathrm{Mpc}^{-1}\right)\right)$, on the basis of the most recent cosmological data [22]. However, for sake of presentation of the results of the present analysis, which, anyway, never entail values of $\Omega_{\chi} h^{2}$ in excess of 0.6 (see last section), we do not impose the bound $\Omega_{C D M} h^{2} \leq 0.3$ in our selection of susy configurations. The neutralino relic abundance is calculated here as illustrated in Ref. [23]. We have checked that susy configurations which could potentially lead to coannihilation effects [24] are marginal in our selected supersymmetric parameter space.

A few comments are in order here. The restrictive assumptions a) - c) adopted above in the framework of the MSSM are instrumental in reducing the otherwise large number of independent parameters to a handful set of them (six in our scheme), and in making the calculations of a number of crucial observables (such as relic abundances and event rates) manageable. The few independent parameters of this simplified MSSM have the role of relevant scales for some fundamental quantities, such as scalar masses and gaugino masses, which in turn determine the size of the numerical outputs. This version of MSSM is obviously the simplest scheme for a susy model, and the most natural one to start with. However, one has to be aware of the fact that new experimental data could eventually force one to adopt more involved versions of supersymmetric models, for instance by relaxing some GUT-inspired relation (such as $M_{1} \simeq 0.5 M_{2}$ ) Ref. [25], or by including $\mathrm{CP}$-violating phases [26].

As regards the distribution of relic neutralinos in our Galaxy, to start with we have assumed a standard halo population with a Maxwellian velocity distribution, whose dispersion speed is centered around $270 \mathrm{~km} \mathrm{~s}^{-1}$ (i.e., $v_{0}=220 \mathrm{~km} \mathrm{~s}^{-1}$ ). However, in the implementation of constraints from up-going muons at neutrino telescopes, we have also considered recent theoretical developments which may have quite contrasting effects on the expected signals [27,28]. These different instances are examined in Sect. III.

Data on antiprotons in space, combined with recent evaluations of the secondary antiproton component in cosmic rays due to spallation processes, are employed in Sect. IV to put further constraints on the original set $S$ of susy configurations, singled out by the DAMA data.

We give the results of our combination of the annual-modulation data with indirect measurement constraints in Sect. V, where we also discuss the cosmological properties for our set of relic neutralinos and present our conclusions. 


\section{SET OF SUPERSYMMETRIC CONFIGURATIONS SINGLED OUT BY THE ANNUAL-MODULATION DATA}

In our papers of Ref. [Q [4] we proved that the DAMA annual-modulation region of Ref. [1] is widely compatible with an interpretation in terms of relic neutralinos, by showing that a sizeable portion of that region is covered by supersymmetric configurations, satisfying all accelerator bounds. Now we show in Fig. 1 that the new, more constrained annual-modulation region of Ref. [6] is still largely compatible with the relic neutralino interpretation, though the supersymmetric space is now more severely constrained by the current limits from accelerators [16, 17].

In deriving the scatter plot shown in Fig. 1 we have used the scan of the susy parameter space defined in the previous section. The neutralino-nucleon cross section has been calculated with the formulae reported in Ref. [2]. As discussed in Ref. [4], this cross section suffers from significant uncertainties in the size of Higgs-quark-quark and squarkquark-neutralino couplings. In fact, these couplings depend on quark masses $m_{q}$ and quark scalar densities in the nucleon $\langle\bar{q} q\rangle$, which are still rather poorly determined. To be specific, we refer to the following quantities: the fractional strange-quark content of the nucleon $y=2<\bar{s} s>/(<\bar{u} u+\bar{d} d>)$, the quark mass ratio $r=2 m_{s} /\left(m_{u}+m_{d}\right)$, and the products $m_{q}<\bar{q} q>$ 's. In our analysis we have taken into account the uncertainties in these quantities. Thus, our scatter plots comprise representative points which have been derived by using both of the two following sets of values, cumulatively:

Set 1: $\quad y=0.33, r=29$,

$m_{l}<\overline{l l}>=23 \mathrm{MeV}, m_{s}<\bar{s} s>=215 \mathrm{MeV}, m_{h}<\bar{h} h>=50 \mathrm{MeV}$.

Set 2: $\quad y=0.50, r=29$,

$$
m_{l}<\bar{l} l>=30 \mathrm{MeV}, m_{s}<\bar{s} s>=435 \mathrm{MeV}, m_{h}<\bar{h} h>=33 \mathrm{MeV} .
$$

In Eqs. (1- 2) $l$ stands for light quarks, $s$ is the strange quark and $h=c, b, t$ denotes heavy quarks. For the light quarks, we have defined $m_{l}<\bar{l} l>\equiv \frac{1}{2}\left[m_{u}<\bar{u} u>+m_{d}<\bar{d} d>\right]$. Set 1 and set 2 bracket, at least partially, the present uncertainties. In Sect. V.B, in connection with neutralino cosmological properties we will also mention the consequences of using a more extreme set of values (set 3 of Ref. [4). For the derivation of the values of the various sets see Ref. [4]. It is worth noticing that a new derivation of the pion-nucleon sigma term, $\sigma_{\pi N}$, points to rather high values: $\sigma_{\pi N}=73.5 \pm 9 \mathrm{MeV}$ [29]. By itself, this new result would increase the value of the quantity $m_{s}<\bar{s} s>$ given in Eq.(2) by $\sim 30 \%$. We recall that the quantity $m_{s}<\bar{s} s>$ is crucial in establishing the size of $\sigma_{\text {scalar }}^{(\text {nucleon })} 30$.

As for the values to be assigned to the quantity $\xi=\rho_{\chi} / \rho_{l}$ we have adopted a standard rescaling recipe. For each point of the parameter space, we take into account the relevant value of the cosmological neutralino relic density. When $\Omega_{\chi} h^{2}$ is larger than a minimal value $\left(\Omega h^{2}\right)_{\min }$, compatible with observational data and with large-scale structure calculations, we simply put $\xi=1$. When $\Omega_{\chi} h^{2}$ turns out to be less than $\left(\Omega h^{2}\right)_{\min }$, and then the neutralino may only provide a fractional contribution to dark matter, we take $\xi=\Omega_{\chi} h^{2} /\left(\Omega h^{2}\right)_{\text {min }}$. The value to be assigned to $\left(\Omega h^{2}\right)_{\min }$ is somewhat arbitrary, in the range $0.01 \lesssim\left(\Omega h^{2}\right)_{\min } \lesssim 0.3$. 
We use here the value $\left(\Omega h^{2}\right)_{\min }=0.01$, which is conservatively derived from the estimate $\Omega_{\text {galactic }} \sim 0.03$.

As we mentioned above, Fig. 1 shows that the annual-modulation region (here depicted for $\rho_{l}=0.3 \mathrm{GeV} \mathrm{cm}^{-3}$ ) is largely covered by the scatter plot. This turns out to be the case also for the other representative values of $\rho_{l}$, as is shown in Fig. 2. In each panel of this figure we only display the portion of the susy scatter plot which is contained in each of the relevant experimental region. In going from the generic scanning used for Fig. 1 to the one employed for Fig. 2, although keeping the overall range of variation of the susy parameter space, we have optimized the numerical scanning in order to have a number of configurations, large enough for our subsequent analyses. The covering by the scatter plots of the annual-modulation regions pertaining to different values of $\rho_{l}$ is more extended for large values of $\rho_{l}$ than for the small ones, as expected from the features of the generic plot of Fig. 1.

We define as set $S$ of susy configurations the set comprised of the configurations whose representative points in the plane $m_{\chi}-\sigma_{\text {scalar }}^{(\text {nucleon })}$ lie inside the annual-modulation regions displayed in Fig. 2. Only configurations of set $S$ are retained in the analyses presented hereafter. We remark that set $S$ is the union of all the subsets of susy configurations which refer to each of the following representative values for $\rho_{l}$ and $v_{0}: \rho_{l}=0.1,0.3,0.5,0.7 \mathrm{GeV}$ $\mathrm{cm}^{-3}, v_{0}=170,220,270 \mathrm{~km} \mathrm{~s}^{-1}$, separately. At any stage, our results will be analysed and presented in our figures in terms of the chosen representative values of $\rho_{l}$ and $v_{0}$, separately.

Another experiment of WIMP direct detection, the CDMS experiment [31], is now entering the DAMA sensitivity region. The current CDMS upper bounds (either with or without subtractions) concern the upper left corner of the annual-modulation regions, which is anyway poorly populated by susy configurations; thus, they are currently marginal in constraining the set $S$. The upper left corner of the annual-modulation regions is also partially disallowed by independent constraints due to indirect measurements (see Sect. V).

Now we turn to the constraints which may be applied to the set $S$, using data from WIMP indirect search experiments. We set the limits for exclusion at the same C.L. to which the DAMA region is currently set, i.e. 99.7\% C.L.

\section{CONSTRAINTS FROM NEUTRALINO-NEUTRALINO ANNIHILATION INSIDE EARTH AND SUN}

Indirect evidence for WIMPs in our halo may be obtained at neutrino telescopes by measurements of the up-going muons, which would be generated by neutrinos produced by pair annihilation of neutralinos captured and accumulated inside the Earth and the Sun 32 34. The size of the expected muon fluxes strongly depends on how these relic particles are distributed in the phase space and on the intrinsic efficiency of the celestial body in capturing the surrounding WIMPs.

In the case of the Sun the capture rate is essentially determined by its strong gravitational field and by the size of the cross section of neutralino scattering off single protons. Instead, in the case of the Earth the capture process may quite significantly be enhanced by coherent neutralino-nucleus cross sections, whose size depends on mass-matching condition between $m_{\chi}$ and the nuclear mass of the dominant chemical constituents of the Earth $(\mathrm{O}, \mathrm{Si}, \mathrm{Mg}$, 
Fe) [35].

As for the phase-space neutralino distribution in our neighbourhood, together with the usual one based on the standard Maxwellian velocity distribution, whose dispersion speed is centered around $270 \mathrm{~km} \mathrm{~s}^{-1}$, we also consider two intriguing and conflicting models which have been recently discussed in the literature.

Damour and Krauss [27] have proposed the existence of a solar-bound population, with velocities restricted to rather low values, $v \lesssim 50 \mathrm{~km} \mathrm{~s}^{-1}$ (for other papers on hypothetical solar-bound WIMP populations, see Refs. [36-39]). The Damour-Krauss solar-bound population would have been produced by WIMPs which scattered off the Sun surface and were set (by perturbations from other planets) into orbits which cross the Earth orbit, but not the Sun. The ensuing velocities would be distributed in the range $25 \mathrm{~km} \mathrm{~s}^{-1} \lesssim v \lesssim 50 \mathrm{~km}$ $\mathrm{s}^{-1}$. This population, although totally irrelevant for the direct measurements by the DAMA $\mathrm{NaI}$-detector, whose electron-equivalent threshold energy is $2 \mathrm{keV}$, has been shown to be potentially important in making the capture of relic neutralinos by the Earth particularly efficient, with a consequent enhancement of the expected output of up-going muons from the Earth, as compared to the standard one [40]. For simple kinematical reasons, the lowerspeed cut off implies that this enhancement is limited to WIMPs of masses lower than $\sim$ $150 \mathrm{GeV}$.

On the other side, Gould and Alam [28], using arguments based on calculations of asteroids trajectories 41], have pointed out that solar-bound WIMPs could evolve in a way quite different from the one derived in Ref. [27], with an ensuing suppression of the up-going muon flux usually expected from the center of the Earth for a standard halo population. This suppression would be significant for WIMP masses above $\sim 65 \mathrm{GeV}$.

In the present paper we take into considerations all of these possible instances. First we consider the standard situation of a Maxwellian velocity distribution over the whole speed range, then we proceed to a critical examination of the other two cases, in which the lowspeed interval is either overpopulated [27] or de-populated [28], as compared to the standard one.

The neutrino spectrum and the ensuing up-going muon flux $\Phi_{\mu}$ are calculated as explained in Refs. [33, 34]. Their normalization is set by the annihilation rate $\Gamma_{A}$ of the neutralinos inside the celestial body (Earth or Sun), and $\Gamma_{A}$ depends, in turn, on the capture rate $C$ of the relic neutralinos by the celestial body through the formula $\Gamma_{A}=\frac{C}{2} \tanh ^{2}\left(\frac{t}{\tau_{A}}\right)$,42, where $t$ is the age of the macroscopic body ( $t \simeq 4.5$ Gyr for Sun, Earth) and $\tau_{A}=\left(C C_{A}\right)^{-1 / 2}$, $C_{A}$ is the annihilation rate proportional to the neutralino-neutralino annihilation crosssection and $C$ denotes the capture rate. In a given macroscopic body the equilibrium between capture and annihilation (i.e. $\Gamma_{A} \sim C / 2$ ) is established only when $t \gtrsim \tau_{A}$. Whereas, in the case of the Sun, the capture-annihilation equilibrium is usually reached, due to the much more efficient capture rate due to the stronger gravitational field, for the Earth, the equilibrium condition is not easily realized.

For the case of the standard halo population with a Maxwellian velocity distribution, $C$ and $\Gamma_{A}$ are calculated as in Refs. [33, 34], and the ensuing muon flux is denoted by $\left(\Phi_{\mu}^{\text {Earth }}\right)^{\text {std }}$. For the Damour-Krauss population the quantities $C$ and $\Gamma_{A}$ are evaluated according to the formulae of Ref. [40] (the relevant muon flux is denoted by $\left.\left(\Phi_{\mu}^{\text {Earth }}\right)^{\mathrm{DK}}\right)$. For the model conjectured by Gould and Alam [28], we have applied to the standard capture rate 
a suppression factor, which we have re-calculated ab initio in the scheme denoted as ultraconservative in Ref. [28], to cover the whole range of masses involved in the present paper. For many susy configurations the suppression factor in the ensuing up-going muon fluxes from the center of the Earth is stronger than the reduction factor in the capture rate alone, due to the relation between $\Gamma_{A}$ and $C$, previously mentioned. For these configurations a reduction in the capture rate induces in the muon flux an extra suppression due to a critical increase in the time required for reaching equilibrium. The muon flux calculated in the Gould-Alam model is denoted here as $\left(\Phi_{\mu}^{\mathrm{Earth}}\right)^{\mathrm{GA}}$.

All our neutrino fluxes include neutrino oscillations and use the procedure outlined in Ref. [43]. Here we assume $\nu_{\mu} \rightarrow \nu_{\tau}$ oscillations, with values for the oscillation parameters which are taken from the best fit performed in Ref. [44] over the whole set of experimental data on atmospheric neutrinos: $\Delta m^{2}=3 \cdot 10^{-3} \mathrm{eV}^{2}, \sin \theta=1$.

Some of our results are presented in Figs. 3-6, where we report various muon fluxes (or ratios of them) versus $m_{\chi}$, for the four representative values of $\rho_{l}$. The solid lines, depicted in Fig. 3 and Fig. 6, denote the 99.7\% C.L. upper bounds, $\left(\Phi_{\mu}^{\text {Earth }}\right)^{l i m}$, derived from the data of the MACRO experiment [45] from the center of the Earth and from the Sun, respectively (for similar limits from the Baksan experiment see Ref. [16]).

The scatter plots of Fig. 3 display some expected characteristic features, such as the peak at $m_{\chi} \sim 50-60 \mathrm{GeV}$, due to the mass-matching between $m_{\chi}$ and $m_{\mathrm{Fe}}$. We notice that a number of configurations induce up-going muon fluxes in excess of the experimental bounds. Figs. 4-5 show what would be the enhancement or the reduction effect in $\Phi_{\mu}^{\text {Earth }}$ in the case of the Damour-Krauss population or in the Gould-Alam conjecture, respectively. The size of these effects agree with the evaluations in Refs. [40,28]. For the Damour-Krauss population, the enhancement effect for some susy configurations appears larger here than in Ref. [40]; this is due to configurations (not considered in [40]) where rescaling in $\rho_{\chi}$ is effective. In Fig. 6 we display the scatter plots for the up-going muon flux from the Sun, expected for the standard halo population. The current experimental bound [45] sets quite marginal constraints.

In Sect. $V$ we use the results of this section to constrain the susy configurations of set $S$. The question, as of which model for the low-speed WIMP population among the two extremes of Refs. [27,28 is applicable, is still open. Thus, we implement here the experimental bounds on the standard flux of up-going muons; namely, we exclude those configurations, whose $\left(\Phi_{\mu}^{\text {Earth }}\right)^{\text {std }}$ is in excess of the $99.7 \%$ C.L. upper bound derived from the MACRO data.

\section{CONSTRAINTS FROM COSMIC-RAY ANTIPROTONS}

The possibility that annihilation of relic particles in the galactic halo might distort the spectrum of cosmic-ray antiprotons at low-kinetic energies $\left(T_{\bar{p}} \lesssim 1 \mathrm{GeV}\right)$ has been considered by many authors 4750 . Indeed, in this energy range, the production of secondary antiprotons by interactions of primary cosmic-ray protons with the interstellar hydrogen has a kinematical drop off [51], which primary $\bar{p}$ 's, created by relic neutralinos of appropriate mass and composition, might fill in, at least partially. The effectiveness of this argument to disentangle ordinary spallation contribution from a possible exotic component due to 
relic particles depends dramatically on how accurately the secondary spectrum is calculated $52,49,50$.

This point was addressed in Ref. [49]. In that paper we improved the evaluation of the energy losses undergone by secondary antiprotons during their diffusion inside the Galaxy, we noticed that the as-yet most recent experimental data (BESS95 [53]) were fitted reasonably well by the secondary spectrum alone, and we examined critically how much room was still available, in the low-energy spectrum, for a contribution from an exotic component. Now, new experimental data (BESS97 [54]) and improved evaluations of the secondary spectrum [50,55,56] further constrain the room left for primary sources. These instances, instrumental in making the separation between primary and secondary antiprotons more difficult, nevertheless confer to the cosmic-ray antiproton measurements a potentially more important role in establishing stringent constraints for relic neutralinos of relatively low mass in our halo, once some of the sizeable, still persisting, uncertainties are reduced.

In the present work we have evaluated the primary antiproton flux, expected from neutralino annihilation, as in Ref. [49], restricting the supersymmetric configurations to those of set $S$. We refer to 49 for all the details concerning the evaluation of the production of these primary antiprotons as well as for the properties related to their propagation in the halo and in the heliosphere. Here we only recall the features of the neutralino mass distribution function adopted in 49] as well as here. This mass distribution function is taken spheroidal and parameterized as a function $\rho_{\chi}(r, z)$ of the radial distance $r$ from the galactic center in the galactic plane and of the vertical distance $z$ from the galactic plane in the form

$$
\rho_{\chi}(r, z)=\rho_{\chi} \frac{a^{2}+r_{\odot}^{2}}{a^{2}+r^{2}+z^{2} / f^{2}},
$$

where $a$ is the core radius of the halo, $r_{\odot}$ is the distance of the Sun from the galactic center and $f$ is a parameter which describes the flattening of the halo. Here we take the values: $a=3.5 \mathrm{kpc}, r_{\odot}=8 \mathrm{kpc}$. In the case of a spherical halo $(f=1)$, we use the value $\rho_{l}=0.3$ $\mathrm{GeV} \mathrm{cm}{ }^{-3}$. When $f<1$ (oblate spheroidal distribution), $\rho_{l}$ is taken as [57, 10]

$$
\rho_{l}(f)=\rho_{l}(f=1) \frac{\sqrt{1-f^{2}}}{f \operatorname{Arcsin} \sqrt{1-f^{2}}} .
$$

For each value of $\rho_{l}$ and of the relevant value of $f: \rho_{l} /\left(\mathrm{GeVcm}^{-3}\right)=0.1(f=1), 0.3$ $(f=1), 0.5(f=0.50), 0.7(f=0.33)$, we have evaluated the top-of-atmosphere (TOA) antiproton fluxes, as the sum of the secondary flux and of the primary flux due to neutralino annihilation for the various supersymmetric configurations of set $S$, pertaining to that specific value of $\rho_{l}$. The secondary flux has been taken from Ref. [55]. Re-acceleration effects in the cosmic rays propagation, which might also be relevant for the features of the secondary antiproton spectrum at low energies [58,56], are not included here. Solar modulation has been evaluated according to the procedure discussed in Ref. [49]. We have compared our theoretical results with the combined experimental data of BESS95 and BESS97 [54], over the whole experimental energy-range $\left(0.18 \mathrm{GeV} \leq T_{\bar{p}} \leq 3.56 \mathrm{GeV}\right)$, by a $\chi^{2}$ calculation.

The results are reported in Fig. 7. In the evaluation of the $\chi^{2}$, in addition to the experimental errors, we have also taken into account the theoretical uncertainties, estimated 
according to the results in Refs. [49,50], with their appropriate energy dependence. Orientatively, they are in the following ranges: $\pm(45-55) \%$ for the primary fluxes, $\pm(60-75) \%$ for the secondaries, depending on the energy bin.

In the following, we adopt the selection criterion of excluding from set $S$ the configuration whose reduced $\chi^{2}$ is above the value $\chi_{r}^{2}=2.44$, which corresponds to a $99.7 \%$ C.L. for the 13 d.o.f. of the BESS $95+97$ data. From Fig. 7 we notice that, especially at large values of $\rho_{l}$, this constraint disallows a number of susy configurations. The reason why the cosmic-ray antiprotons constraint is not more effective in constraining set $S$ is to be attributed mainly to the current large uncertainties affecting the evaluation of antiproton propagation in the galactic halo and in the heliosphere.

\section{RESULTS AND CONCLUSIONS}

Now we apply the experimental bounds from indirect searches discussed in Sects. III-IV to constrain the supersymmetric configurations of set $S$.

\section{A. Combining direct and indirect measurements}

Fig. 8 displays the extent of the covering of the annual-modulation regions (one for each value of $\rho_{l}$ ) by the susy configurations, when the MACRO upper bounds are applied to $\left(\Phi_{\mu}^{\text {Earth }}\right)^{\text {std }}$. A comparison of this figure with Fig. 2 shows that the implementation of these limits somewhat de-populate the covering regions, with a marked effect for the value of the neutralino mass which matches the mass of Iron, as expected. Apart from this, the extent of the regions covered by the scatter plots does not significantly change.

Fig. 9 depicts what would be the effect for a solar-bound WIMP population à la DamourKrauss. Especially at low values of $\rho_{l}$ there would be some shrinking of the original regions of the scatter plots in their upper parts, but still the annual-modulation regions would be widely covered by physical susy configurations. At variance with this case, the Gould-Alam conjecture would relax the consequences of the constraints applied in obtaining the plots of Fig. 8.

Now, we return to the case where the experimental bounds $\left(\Phi_{\mu}^{\text {Earth }}\right)^{l i m}$ are applied on $\left(\Phi_{\mu}^{\text {Earth }}\right)^{\text {std }}$. When, on top of these constraints, we also implement the constraints due to cosmic-ray antiprotons, we obtain that the scatter plots of Fig. 8 become somewhat depopulated, but without any appreciable modification in the contours of the covering regions, except for a quite marginal downward shift in their upper-left parts. Therefore Fig. 8 may be considered as the final situation of our analysis, once also the implementation of the antiprotons constraints has been applied. We denote as set $T$ the subset of $S$ which comprises the susy configurations not disallowed by bounds on the standard up-going muon fluxes and on cosmic-ray antiprotons.

We have analyzed the main properties of the configurations of set $T$; some of them are displayed in Figs. 10-11. We recall that the scatter plots of these figures are derived, as all previous ones, by using for the hadronic quantities, discussed in Sect. II, set 1 and set 2, cumulatively. In Fig. 10 we note that the configurations of set $T$ cover only a specific region of the susy parameter space not yet disallowed by accelerator constraints. The shape 
of the distribution of the representative points of $T$ in the plot of Fig. 10 is simply explained by the fact that the values of the scalar neutralino-nucleon cross section at the level of the DAMA data require either a large $\tan \beta$ or a small $m_{h}$ (or both of these two conditions). This constraint is stronger when the values of the hadronic quantities are restricted to set 1 , alone. Fig. 11 displays a correlation among $m_{A}$ and $m_{0}$ which is mainly due to the interplay of these two quantities in generating a light $m_{h}$. Again, restricting the scatter plot to points belonging to set 1 , this correlation becomes more pronounced. We recall that, at variance with constrained sugra-supersymmetric models, in the MSSM we are using here, $m_{A}$ and $m_{0}$ are treated as independent parameters.

\section{B. Cosmological properties}

We turn now to an analysis of the cosmological properties of relic neutralinos of the susy configurations of set $T$. The relevant plots $\Omega_{\chi} h^{2}$ vs $m_{\chi}$ are displayed in Fig. 12. It is remarkable that the region of main cosmological interest: $\Omega_{\chi} h^{2} \gtrsim 0.03$ [22] turns out to be widely populated, with values of $\Omega_{\chi} h^{2}$ which approach, and even exceed, what may be considered as the current upper bound for cold dark matter: $\Omega_{C D M} h^{2} \lesssim 0.3$ [22]. This means that the DAMA annual-modulation data are compatible with a neutralino as a major component of dark matter. We stress that the scatter plot would even shift upward, should we use for the hadronic quantities discussed in Sect. II the following set: $y=0.50, r=36$, $m_{l}<\bar{l} l>=33 \mathrm{MeV}, m_{s}<\bar{s} s>=585 \mathrm{MeV}, m_{h}<\bar{h} h>=21 \mathrm{MeV}$. This set of values, denoted as set 3 in Ref. [4], is more extreme as compared to set 1 and set 2, but still compatible with the current uncertainties.

Finally, we notice that a rather strong de-population in the plots of Fig. 12 is present around $\Omega_{\chi} h^{2} \simeq 0.01$ and for large values of $\rho_{l}$. This effect is induced by the cosmic-ray antiproton constraint, since the calculated $\bar{p}$ fluxes have their maximal values for $\Omega_{\chi} h^{2}$ close to the value below which we apply the rescaling of the local density, i.e. $\left(\Omega h^{2}\right)_{\min }=0.01$. This property is quite general in this class of calculations, and it was already commented upon, for instance, in Ref. [2].

\section{Conclusions}

In the present paper we have examined the possibility that the annual-modulation effect, measured by the DAMA Collaboration at a $4 \sigma$ confidence level [6], may be interpreted in terms of relic neutralinos. We have examined this problem, by employing the Minimal Supersymmetric extension of the Standard Model, as a model which does not impose too strong theoretical prejudices on the phenomenological analysis. We have taken into account all experimental constraints, from accelerators and from WIMP indirect experiments.

Let us now summarize our main conclusions:

- The annual-modulation effect mentioned above turns out to be compatible with an interpretation in terms of relic neutralinos. 
- The set of supersymmetric configurations selected by the annual-modulation data is only modestly reduced by current experimental data from WIMP indirect searches (up-going muons from the Earth and the Sun, and cosmic--ray antiprotons).

- The set of supersymmetric configurations, selected by the annual-modulation data and not disallowed by the indirect measurements, comprise configurations of relevant cosmological interest, with relic neutralinos playing the role of a major dark matter constituent.

The phenomenological analysis presented in this paper goes beyond the discussion of the experimental data specifically discussed here. We have tried to pin down the most relevant theoretical points, which are still at the origin of large uncertainties, and then require additional investigation. These are: i) size of the Higgs-quark-quark and the squarkquark-neutralino couplings, ii) properties of the WIMP distribution at low velocities (with the possible existence of a solar-bound WIMP population), iii) accurate determination of the propagation in the galactic halo and in the heliosphere for cosmic-ray antiprotons.

\section{ACKNOWLEDGMENTS}

This work was partially supported by the Research Grants of the Italian Ministero dell'Università e della Ricerca Scientifica e Tecnologica (MURST) within the Astroparti-

cle Physics Project, by the Spanish DGICYT under grant number PB98-0693, and by the TMR network grant ERBFMRXCT960090 of the European Union. 


\section{REFERENCES}

[1] R. Bernabei et al., Phys. Lett. B424 (1998) 195; Phys. Lett. B450 (1999) 448.

[2] A. Bottino, F. Donato, N. Fornengo and S. Scopel, Phys. Lett.B423 (1998) 109; Phys. Rev. D59 (1999) 095003; Phys. Rev. D59 (1999) 095004; Astrop. Phys. 10 (1999) 203.

[3] P. Belli, R. Bernabei, A. Bottino, F. Donato, N. Fornengo, D. Prosperi and S. Scopel, Phys. Rev. D 61 (2000) 023512.

[4] A. Bottino, F. Donato, N. Fornengo and S. Scopel, hep-ph/9909228 (to appear in Astrop. Phys.).

[5] An analysis of the DAMA annual-modulation data of Refs. [1] in supergravity unified models has also been carried out in R. Arnowitt and P. Nath, Phys. Rev. D 60 (1999) 044002.

[6] R. Bernabei et al., preprint ROM2F/2000/01 (University of Roma at Tor Vergata).

[7] R. Bernabei et al., Phys. Lett. B 389 (1996) 757.

[8] F. Donato, N. Fornengo and S. Scopel, Astrop. Phys. 9 (1998) 297.

[9] We thank the DAMA Collaboration for providing these curves.

[10] E. Gates, G. Gyuk and M.S. Turner, Phys. Rev. Lett. 74, 3724 (1995); Astrophys. J. Lett. 449, L123 (1995); Phys. Rev. D53, 4138 (1996).

[11] E. Gates, G. Gyuk and M.S. Turner, Proceedings of the 18th Texas Symposium on Relativistic Astrophysics, edited by A. Olinto, J. Frieman and D. Schramm (World Scientific, 1998).

[12] H.P. Nilles, Phys. Rep. 110, 1 (1984); H.E. Haber and G.L. Kane, Phys. Rep. 117, 75 (1985); R. Barbieri, Riv. Nuovo Cim. 11, 1 (1988).

[13] A. Bottino and N. Fornengo, hep-ph/9904469, Proceedings of the 5th School on NonAccelerator Particle Astrophysics, Trieste, 29 June-10 July 1998, Eds. R.A. Carrigan, Jr., G. Giacomelli, N. Paver, E.U.T., Trieste, 1999, page 241.

[14] M. Carena, M. Quirós and C.E.M. Wagner, Nucl. Phys. B 416 (1996) 407; H.E. Haber, Z. Phys. C 5 (1996) 1.

[15] V. Berezinsky, A. Bottino, J. Ellis, N. Fornengo, G. Mignola and S. Scopel, Astropart. Phys. 5, 1 (1996).

[16] G. GANIS (LEPSUSY-WG), LEPC, 7 September 1999;

http://www.cern.ch/Committees/LEPC/minutes/LEPC52.html;

T. Alderweireld, I. Gil, P. Rebecchi for DELPHI Collaboration, http://delphiwww.cern.ch/ pubxx/www/delsec/conferences/tampere99/.

[17] J.A.Valls (CDF Coll.) FERMILAB-Conf-99/263-E CDF; http://fnalpubs.fnal.gov/archive/1999/conf/Conf-99-263-E.html

[18] S. Bertolini, F. Borzumati, A. Masiero and G. Ridolfi, Nucl. Phys. B353, 591 (1991); R. Barbieri and G.F. Giudice, Phys. Lett. B309, 86 (1993); R. Garisto and J.N. Ng, Phys. Lett. B315, 372 (1993); F.M. Borzumati, M. Drees and M.M. Nojiri, Phys. Rev. D51, 341 (1995); J. Wu, R. Arnowitt and P. Nath, Phys. Rev. D51, 1371 (1995); V. Barger, M.S. Berger, P. Ohmann and R.J.N. Phillips, Phys. Rev. D51, 2438 (1995).

[19] K. Chetyrkin, M. Misiak and M. Münz, Phys. Lett. B400, 206 (1997); M. Ciuchini, G. Degrassi, P. Gambino and G.F. Giudice, CERN preprint CERN-TH/97-279, 1998, hep-ph/9710335; A. Czarnecki and W.J. Marciano, Brookhaven National Laboratory 
preprint BNL-HET-98/11, 1998, hep-ph/9804252; M. Ciuchini, G. Degrassi, P. Gambino and G.F. Giudice, CERN preprint CERN-TH/98-177, 1998, hep-ph/9806308.

[20] S. Ahmed et al, CLEO Collaboration, CLEO CONF 99-10.

[21] R. Barate et al. (ALEPH Collaboration), Phys. Lett. B 429 (1998) 169.

[22] For comprehensive analyses of the current cosmological data see, for instance: N.A. Bahcall, J.P. Ostriker, S. Perlmutter and P.J. Steinhardt, Science 284 (1999) 1481; C.H. Lineweaver, astro-ph/9911294; J.R. Primack, astro-ph/9912089; M.S. Turner, astroph/9912211.

[23] A. Bottino, V. de Alfaro, N. Fornengo, G. Mignola and M. Pignone, Astropart. Phys. 2, 67 (1994).

[24] P. Binétruy, G. Girardi and P. Salati, Nucl. Phys. B237, 285 (1984); K. Griest and D. Seckel, Phys. Rev. D43, 3191 (1991); S. Mizuta and M. Yamaguchi, Phys. Lett. B298, 120 (1993); J. Edsjö and P. Gondolo, Phys. Rev. D56, 1879 (1997); J. Ellis, T. Falk, K. A. Olive and M. Srednicki, hep-ph/9905481, to appear in Astropart. Phys.

[25] K. Griest and L. Roszkowski, Phys. Rev. D 46 (1992) 3309; S. Mizuta, D. Ng and M. Yamaguchi, Phys. Lett. B 300 (1993) 96.

[26] S. Khalil and Q. Shafi, Nucl. Phys. B 564 (1999) 19; T. Falk, A. Ferstl and K.A. Olive, hep-ph/9908311; P. Gondolo and K. Freese, hep-ph/9908390; S.Y. Choi, S. Khalil, hep-ph/9908397; S. Khalil, hep-ph/9910408; E. Accomando, R. Arnowitt, B. Dutta, hep-ph/0001121; and references quoted therein.

[27] T. Damour and L.M. Krauss, Phys. Rev. Lett. 81 (1998) 5726 and Phys. Rev. D 59 (1999) 063509-1.

[28] A. Gould and S.M.K. Alam, astro-ph/9911288.

[29] M.G. Olsson, hep-ph/0001203.

[30] G.B. Gelmini, P. Gondolo and E. Roulet, Nucl. Phys. B 351 (1991) 623.

[31] D. Bauer (CDMS Collaboration), talk given at PASCOS 99, December 1999.

[32] J. Silk, K. Olive and M. Srednicki, Phys. Rev. Lett. 55 (1985) 257; T. Gaisser, G. Steigman and S. Tilav, Phys. Rev. D34 (1986) 2206; K. Freese, Phys. Lett. B167 (1986) 295; K. Griest and S. Seckel, Nucl. Phys. B279 (1987) 804; G.F. Giudice and E. Roulet, Nucl. Phys. B316 (1989) 429; G.B. Gelmini, P. Gondolo and E. Roulet, Nucl. Phys. B351 (1991) 623; M. Kamionkowski, Phys. Rev. D44 (1991) 3021; A. Bottino, V. de Alfaro, N. Fornengo, G. Mignola and M. Pignone, Phys. Lett. B265 (1991) 57; F. Halzen, M. Kamionkowski and T. Steltzer, Phys. Rev. D45 (1992) 4439; V.S. Berezinsky, Nucl. Phys. (Proc. Suppl.) B31 (1993) 413 (Proc. Neutrino 92, Ed. A. Morales) M. Mori et al., Phys. Rev. D48 (1993) 5505; M. Drees, G. Jungman, M. Kamionkowski and M.M. Nojiri, Phys. Rev. D49 (1994) 636; R. Gandhi, J.L. Lopez, D.V. Nanopoulos, K. Yuan and A. Zichichi, Phys. Rev. D49 (1994) 3691; L. Bergström, J. Edsjö and P. Gondolo, Phys. Rev. D55 (1997) 1765. L. Bergström, J. Edsjö and M. Kamionkowski, Astrop. Phys. 7 (1997) 147.

[33] A. Bottino, N. Fornengo, G. Mignola and L. Moscoso, Astroparticle Physics 3 (1995) 65.

[34] V. Berezinsky, A. Bottino, J. Ellis, N. Fornengo, G. Mignola and S. Scopel, Astrop. Phys. 5 (1966) 333.

[35] A. Gould, Astrophys. J. 321 (1987) 571.

[36] G. Steigman, C.L. Sarazin, H. Quintana and J. Faulkner, Astrophys. J. 83 (1978) 1050. 
[37] K. Griest, Phys. Rev. D 37 (1988) 2703.

[38] A. Gould, J.A. Frieman and K. Freese, Phys. rev. D 39 (1989) 1029.

[39] J.I. Collar, Phys. Rev. D 59 (1999) 063514.

[40] L. Bergström, T. Damour, J. Edsjö, L.M. Krauss and P. Ullio, JHEP 9908 (1999) 010.

[41] P. Farinella et al., Nature 371 (1994) 314; B. Gladman et al., Science 277 (1997) 197; F. Migliorini, P. Michel, A. Morbidelli, D. Nesvorný and V. Zappalà, Science 281 (1998) 2022 .

[42] K. Griest and D. Seckel, Nucl. Phys. B 283 (1987) 681.

[43] N. Fornengo, Proceedings of WIN99, Cape Town, South Africa, January 1999 (Eds. C.A. Dominguez and R.D. Viollier), hep-ph/9904351.

[44] N. Fornengo, M.C. Gonzalez-Garcia, J.W.F. Valle, in preparation.

[45] M. Ambrosio et al., Phys. rev. D 60 (1999) 082002.

[46] O. Suvorova, hep-ph/9911415.

[47] J. Silk and M. Srednicki, Phys. Rev. Lett. 53, 624 (1984); J. Ellis, R.A. Flores, K. Freese, S. Ritz, D. Seckel and J. Silk, Phys. Lett. B214, 403 (1988); F. Stecker, S. Rudaz and T. Walsch, Phys. Rev. Lett. 55, 2622 (1985); J.S. Hagelin and G.L. Kane, Nucl. Phys. B263, 399 (1986); S. Rudaz and F.W. Stecker, Astrophys. J. 325, 16 (1988); F. Stecker and A. Tylka, Astrophys. J. 336, L51 (1989); G. Jungman and M. Kamionkowski, Phys. Rev. D49, 2316 (1994); T. Mitsui, K. Maki and S. Orito, Phys. Lett. B389, 169 (1996).

[48] A. Bottino, C. Favero, N. Fornengo and G. Mignola, Astropart. Phys. 3, 77 (1995).

[49] A. Bottino, F. Donato, N. Fornengo and P. Salati, Phys. Rev. D 58 (1998) 123503.

[50] L. Bergström, J. Edsjö and P. Ullio, astro-ph/9902012.

[51] See, for instance, T.K. Gaisser, Cosmic Rays and Particle Physics (Cambridge University Press, Cambridge, England, 1992).

[52] W.R. Webber, M.S. Poitgeiter, Ap.J. 344 (1989) 779; T.K. Gaisser, R. K. Schaefer, Ap.J. 394 (1992) 174.

[53] H. Matsunaga et al., Phys. Rev. Lett. 81 (1998) 4052.

[54] S. Orito et al., astro-ph/9906426.

[55] J.W. Bieber, R.A. Burger, R. Engel, T.K. Gaisser, S. Roesler and T. Stanev, Phys. Rev. Lett. 83 (1999) 674.

[56] D. Maurin et al., in preparation

[57] J. Binney and S. Tremaine, Galactic Dynamics (Princeton University Press, 1987).

[58] M. Simon and U. Einbach, Ap. J. 456 (1996) 519; A.W. Strong and I.V. Moskalenko, Ap. J. 509 (1998) 212. 


\section{FIGURE CAPTIONS}

FIG. 1. Plot of $\xi \sigma_{\text {scalar }}^{\text {(nucleon) }}$ versus $m_{\chi}$. The solid line delimits the $3 \sigma$ C.L. annualmodulation region, obtained by the DAMA $\mathrm{NaI}(\mathrm{Tl})$ experiment with a total exposure of $57986 \mathrm{~kg}$ - day [6]. This region was obtained by including the upper-bound constraints of Ref. [7], by setting $\rho_{l}$ at the standard reference value: $\rho_{l}=0.3 \mathrm{GeV} \mathrm{cm}^{-3}$, and by taking into account uncertainties in the astrophysical velocities of the usual galactic Maxwellian distribution. Also shown in the present figure are the contour lines for the three values $v_{0}=$ $170 \mathrm{~km} \mathrm{~s}^{-1}$ (short-dashed (red) line), $v_{0}=220 \mathrm{~km} \mathrm{~s}^{-1}$ (long-dash-short-dashed (blue) line), $v_{0}=270 \mathrm{~km} \mathrm{~s}^{-1}$ (long-dashed (green) line), separately. The scatter plot is calculated in the MSSM with the scan described in Sect.I; the points of the scatter plot are coded according to the value of the relic abundance, $\Omega_{\chi} h^{2}$, of the relevant susy configuration: dots denote $\Omega_{\chi} h^{2}<0.01$, crosses denote $0.01<\Omega_{\chi} h^{2}<0.1$ and empty circles denote $\Omega_{\chi} h^{2}>0.1$.

FIG. 2. Location of the DAMA annual-modulation region for four representative values of $\rho_{l}: \rho_{l}=0.1,0.3,0.5,0.7 \mathrm{GeV} \mathrm{cm}^{-3}$. The scatter plots show only the configurations which lie inside the relevant annual-modulation region. A grey-level (color) code is used depending on the value of $v_{0}$ employed in the extraction of the annual-modulation region: medium grey (red) denotes points which lie in the annual-modulation region extracted by setting $v_{0}=170 \mathrm{Km} \mathrm{s}^{-1}$, dark grey (blue) denotes points which lie in the annual-modulation region extracted by setting $v_{0}=220 \mathrm{Km} \mathrm{s}^{-1}$, light grey (green) denotes points which lie in the annual-modulation region extracted by setting $v_{0}=270 \mathrm{Km} \mathrm{s}^{-1}$. The three sets are superimposed in that sequential order.

FIG. 3. Scatter plot for the up-going muon flux from the center of the Earth for a standard Maxwellian distribution, $\left(\Phi_{\mu}^{\text {Earth }}\right)^{\text {std }}$, versus $m_{\chi}$. The grey-level (color) code is the same as in Fig. 2. The solid line denotes the $99.7 \%$ C.L. upper bounds, $\left(\Phi_{\mu}^{\text {Earth }}\right)^{l i m}$, derived from the data of the MACRO experiment [45].

FIG. 4. Enhancement effect in the up-going muon flux from the center of the Earth in case of a solar-bound population à la Damour-Krauss [27]. The grey-level (color) code is the same as in Fig. 2.

FIG. 5. Suppression effect in the up-going muon flux from the center of the Earth in case of the Gould-Alam conjecture [28]. The grey-level (color) code is the same as in Fig. 2 .

FIG. 6. Scatter plot for the up-going muon flux from the Sun for a standard Maxwellian distribution, $\Phi_{\mu}^{\text {Sun }}$, versus $m_{\chi}$. The grey-level (color) code is the same as in Fig. 2. The solid line denotes the $99.7 \%$ C.L. upper bounds, derived from the data of the MACRO experiment [45]. 
FIG. 7. Scatter plot for the reduced $\chi_{r}^{2}$ 's in a comparison of the calculated cosmic-ray antiprotons fluxes with the combined experimental data of BESS95 and BESS97 [54]. The horizontal line denotes the value $\chi_{r}^{2}=2.44$, which for 13 d.o.f. corresponds to a $99.7 \%$ C.L., above which we disallow susy configurations. The grey-level (color) code is the same as in Fig. 2.

FIG. 8. As in Fig. 2, once the constraints from the up-going muon fluxes from the center of the Earth are applied, assuming a Maxwellian halo distribution for relic neutralinos. The grey-level (color) code is the same as in Fig. 2.

FIG. 9. Covering of the annual-modulation regions, if the constraint $\left(\Phi_{\mu}^{\text {Earth }}\right)^{\mathrm{DK}} \leq$ $\left(\Phi_{\mu}^{\text {Earth }}\right)^{\lim }$ were applied. The grey-level (color) code is the same as in Fig. 2.

FIG. 10. Scatter plot for set $T$ in the plane $m_{h}-\tan \beta$. The grey-level (color) code is the same as in Fig. 2. For each panel, the lower dashed line denotes the frontier of the complete scatter plot; the upper dashed line denotes the frontier, when only set 1 for the hadronic quantities of Sect. II is employed. The hatched region on the right is excluded by theory. The hatched region on the left is excluded by present data from LEP I16 and CDF [17]. The solid line represents the 95\% C.L. bound reachable at LEP2, in case of non discovery of a neutral Higgs boson.

FIG. 11. Scatter plot for set $T$ in the plane $m_{0}-m_{A}$. The grey-level (color) code is the same as in Fig. 2. For each panel, the upper dashed line denotes the frontier of the complete scatter plot; the lower dashed line denotes the frontier, when only set 1 for the hadronic quantities of Sect. II is employed.

FIG. 12. Neutralino relic abundance $\Omega_{\chi} h^{2}$ versus $m_{\chi}$, once the constraints from up-going muon fluxes and cosmic-ray antiprotons are applied. The hatched region is disallowed by the upper limit on cold dark matter $\Omega_{C D M} h^{2} \lesssim 0.3[22]$. 


\section{FIGURES}

A. Bottino, F. Donato, N. Fornengo, S. Scopel (2000)

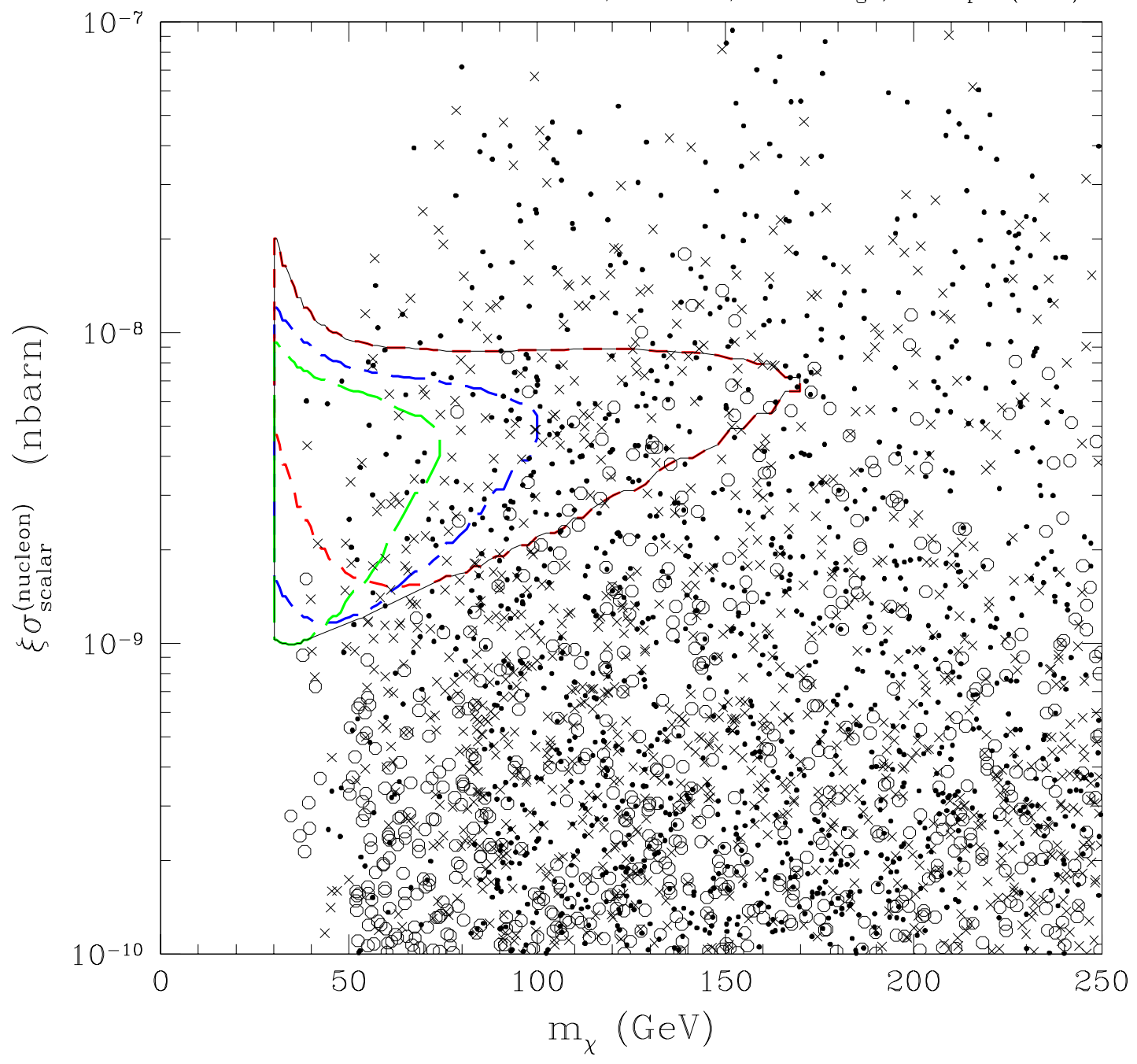

FIG. 1. Plot of $\xi \sigma_{\text {scalar }}^{\text {(nucleon) }}$ versus $m_{\chi}$. The solid line delimits the $3 \sigma$ C.L. annualmodulation region, obtained by the DAMA $\mathrm{NaI}(\mathrm{Tl})$ experiment with a total exposure of $57986 \mathrm{~kg}$ - day [6]. This region was obtained by including the upper-bound constraints of Ref. [7], by setting $\rho_{l}$ at the standard reference value: $\rho_{l}=0.3 \mathrm{GeV} \mathrm{cm}^{-3}$, and by taking into account uncertainties in the astrophysical velocities of the usual galactic Maxwellian distribution. Also shown in the present figure are the contour lines for the three values $v_{0}=$ $170 \mathrm{~km} \mathrm{~s}^{-1}$ (short-dashed (red) line), $v_{0}=220 \mathrm{~km} \mathrm{~s}^{-1}$ (long-dash-short-dashed (blue) line), $v_{0}=270 \mathrm{~km} \mathrm{~s}^{-1}$ (long-dashed (green) line), separately. The scatter plot is calculated in the MSSM with the scan described in Sect.I; the points of the scatter plot are coded according to the value of the relic abundance, $\Omega_{\chi} h^{2}$, of the relevant susy configuration: dots denote $\Omega_{\chi} h^{2}<0.01$, crosses denote $0.01<\Omega_{\chi} h^{2}<0.1$ and empty circles denote $\Omega_{\chi} h^{2}>0.1$. 

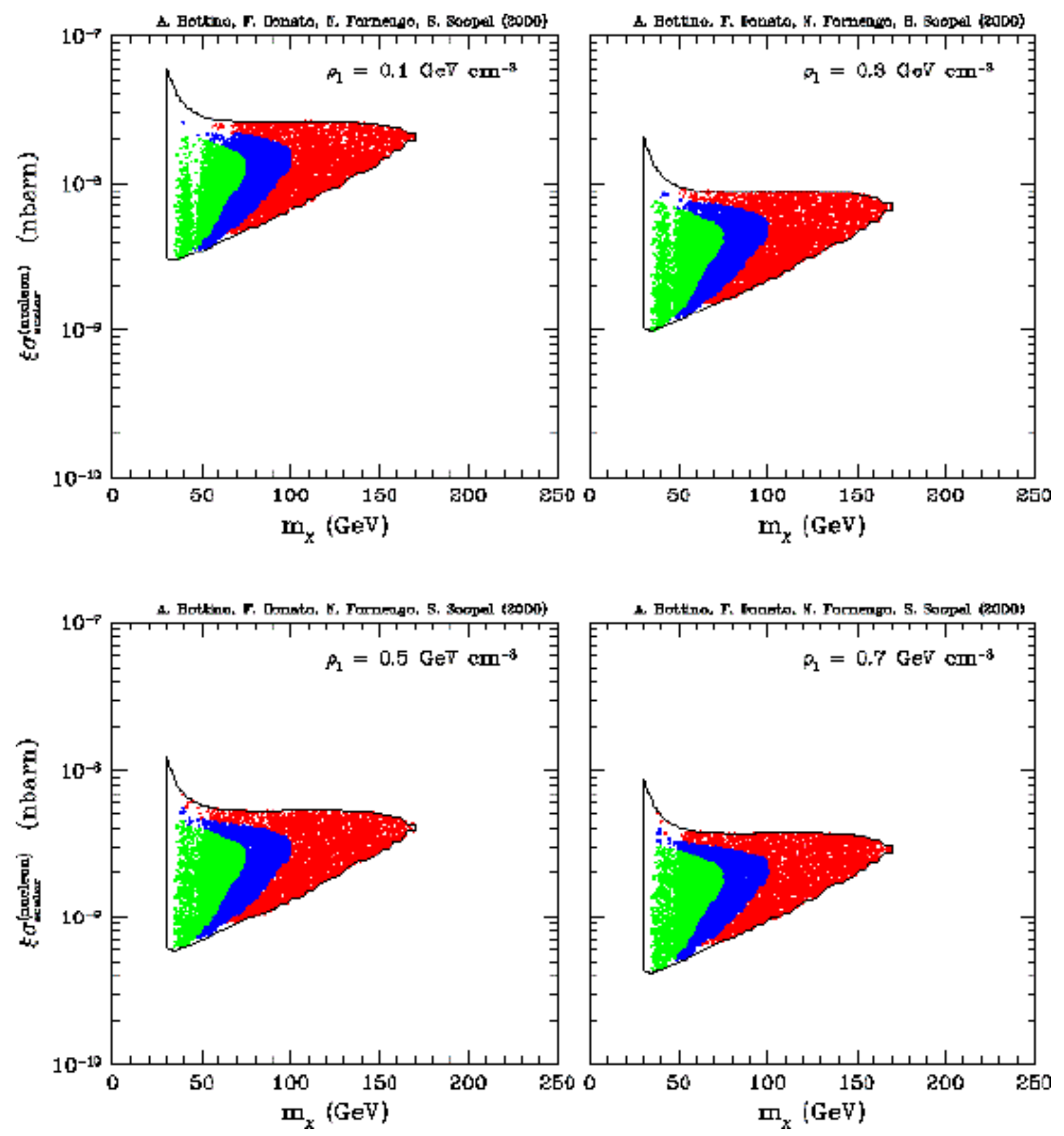

FIG. 2. Location of the DAMA annual-modulation region for four representative values of $\rho_{l}: \rho_{l}=0.1,0.3,0.5,0.7 \mathrm{GeV} \mathrm{cm}^{-3}$. The scatter plots show only the configurations which lie inside the relevant annual-modulation region. A grey-level (color) code is used depending on the value of $v_{0}$ employed in the extraction of the annual-modulation region: medium grey (red) denotes points which lie in the annual-modulation region extracted by setting $v_{0}=170 \mathrm{Km} \mathrm{s}^{-1}$, dark grey (blue) denotes points which lie in the annual-modulation region extracted by setting $v_{0}=220 \mathrm{Km} \mathrm{s}^{-1}$, light grey (green) denotes points which lie in the annual-modulation region extracted by setting $v_{0}=270 \mathrm{Km} \mathrm{s}^{-1}$. The three sets are superimposed in that sequential order. 

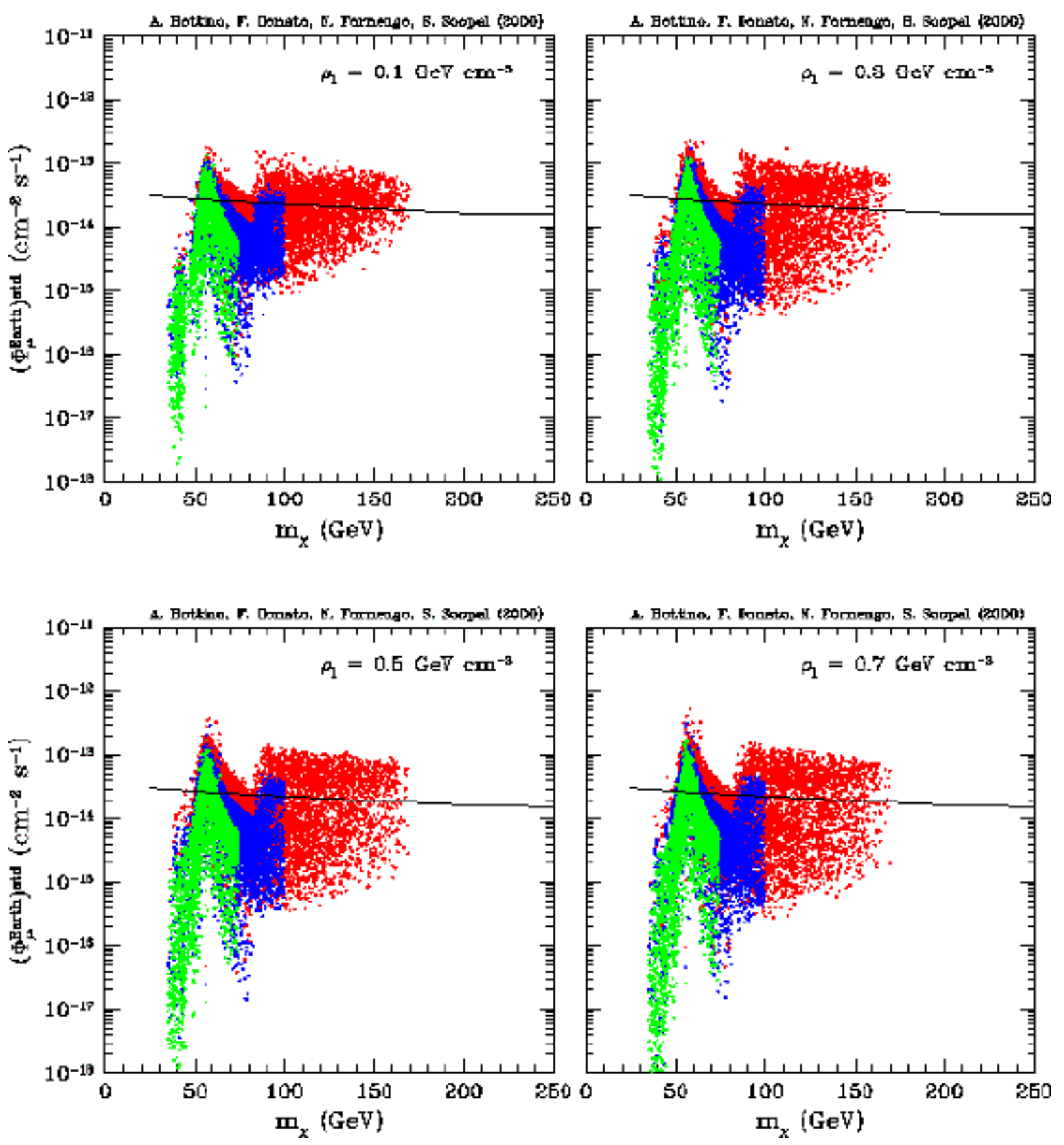

FIG. 3. Scatter plot for the up-going muon flux from the center of the Earth for a standard Maxwellian distribution, $\left(\Phi_{\mu}^{\text {Earth }}\right)^{\text {std }}$, versus $m_{\chi}$. The grey-level (color) code is the same as in Fig. 2. The solid line denotes the $99.7 \%$ C.L. upper bounds, $\left(\Phi_{\mu}^{\text {Earth }}\right)^{\text {lim }}$, derived from the data of the MACRO experiment 45]. 

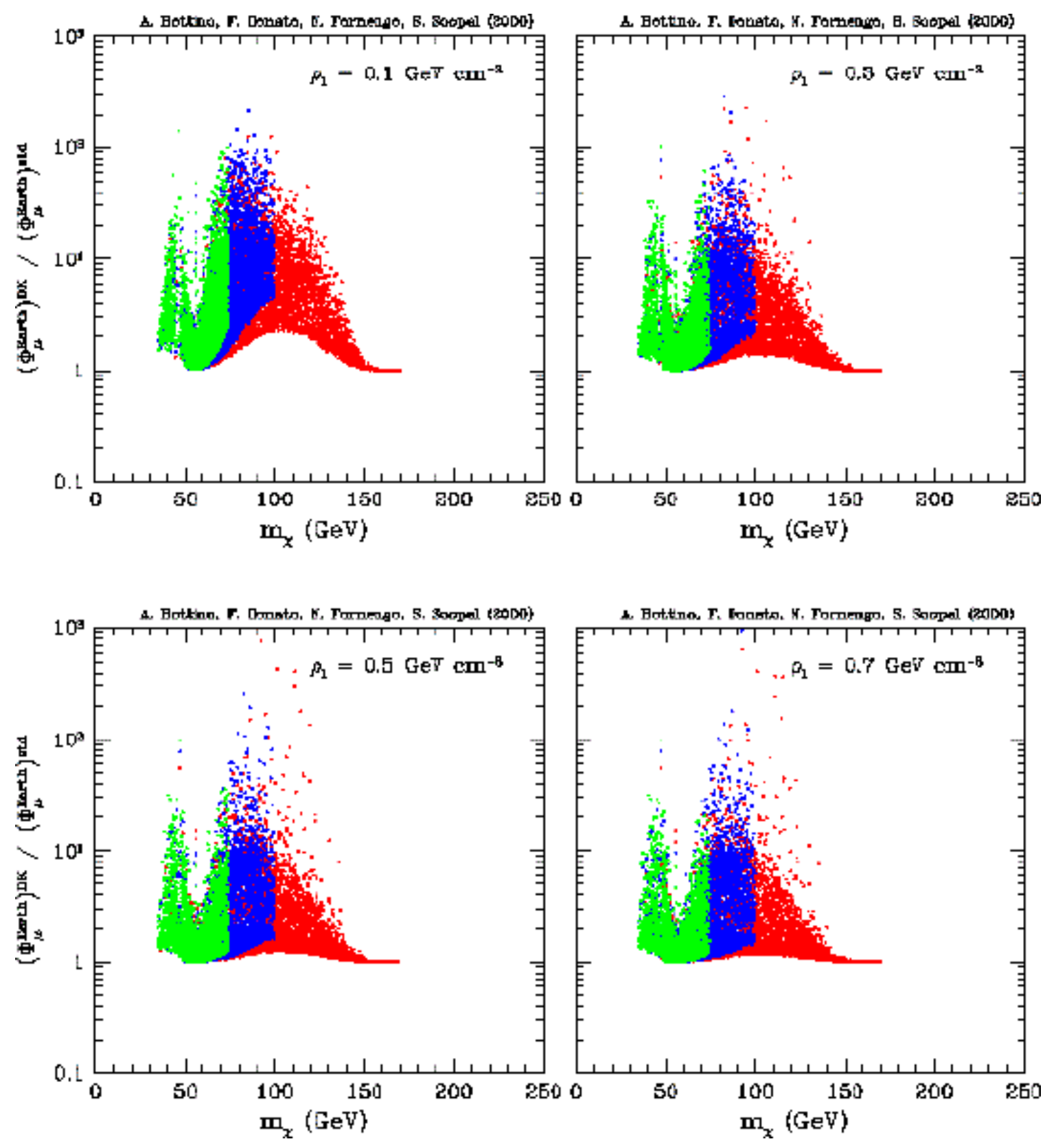

FIG. 4. Enhancement effect in the up-going muon flux from the center of the Earth in case of a solar-bound population à la Damour-Krauss [27]. The grey-level (color) code is the same as in Fig. 2. 

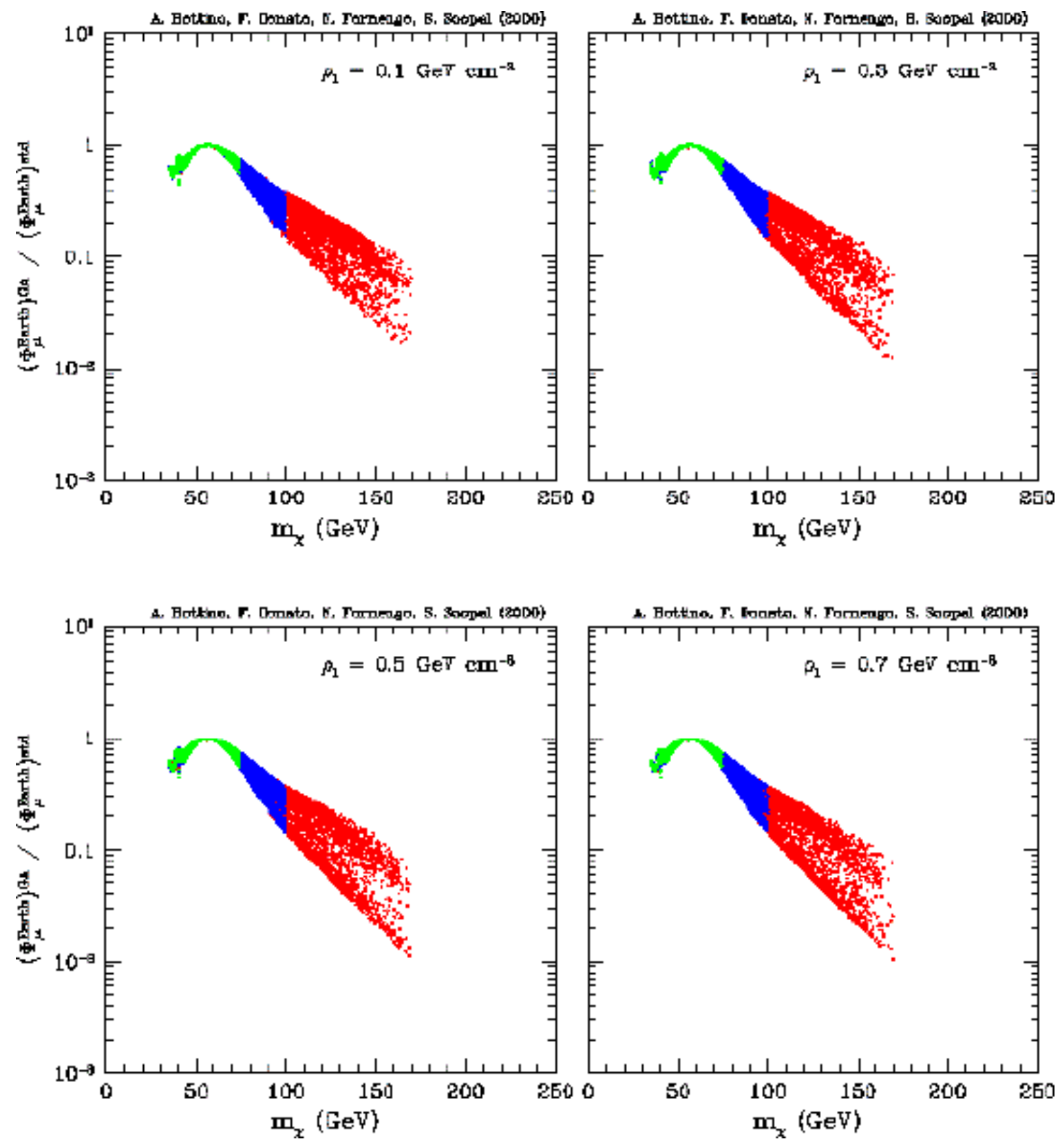

FIG. 5. Suppression effect in the up-going muon flux from the center of the Earth in case of the Gould-Alam conjecture [28]. The grey-level (color) code is the same as in Fig. 2 . 

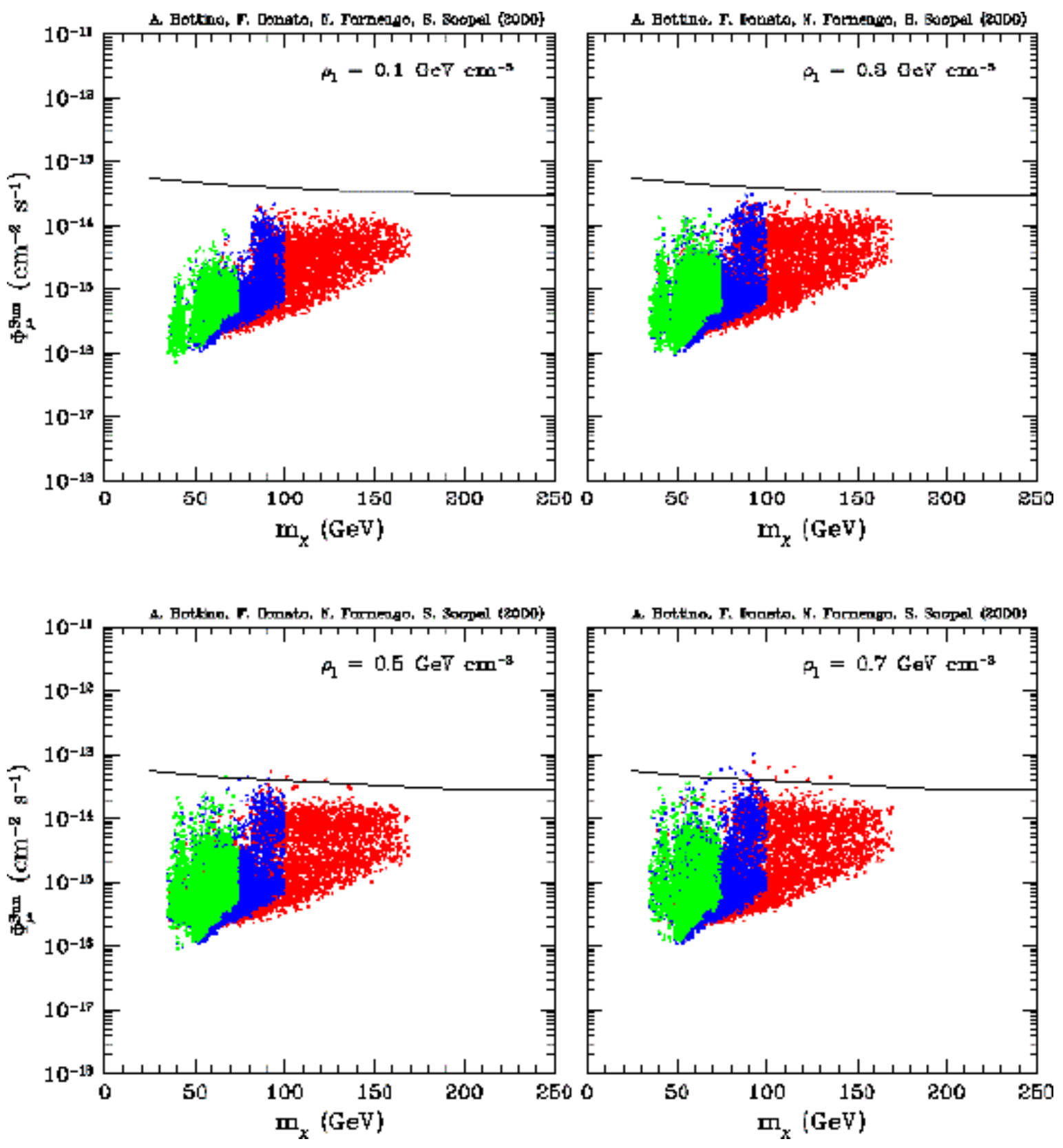

FIG. 6. Scatter plot for the up-going muon flux from the Sun for a standard Maxwellian distribution, $\Phi_{\mu}^{\text {Sun }}$, versus $m_{\chi}$. The grey-level (color) code is the same as in Fig. 2. The solid line denotes the $99.7 \%$ C.L. upper bounds, derived from the data of the MACRO experiment 45. 

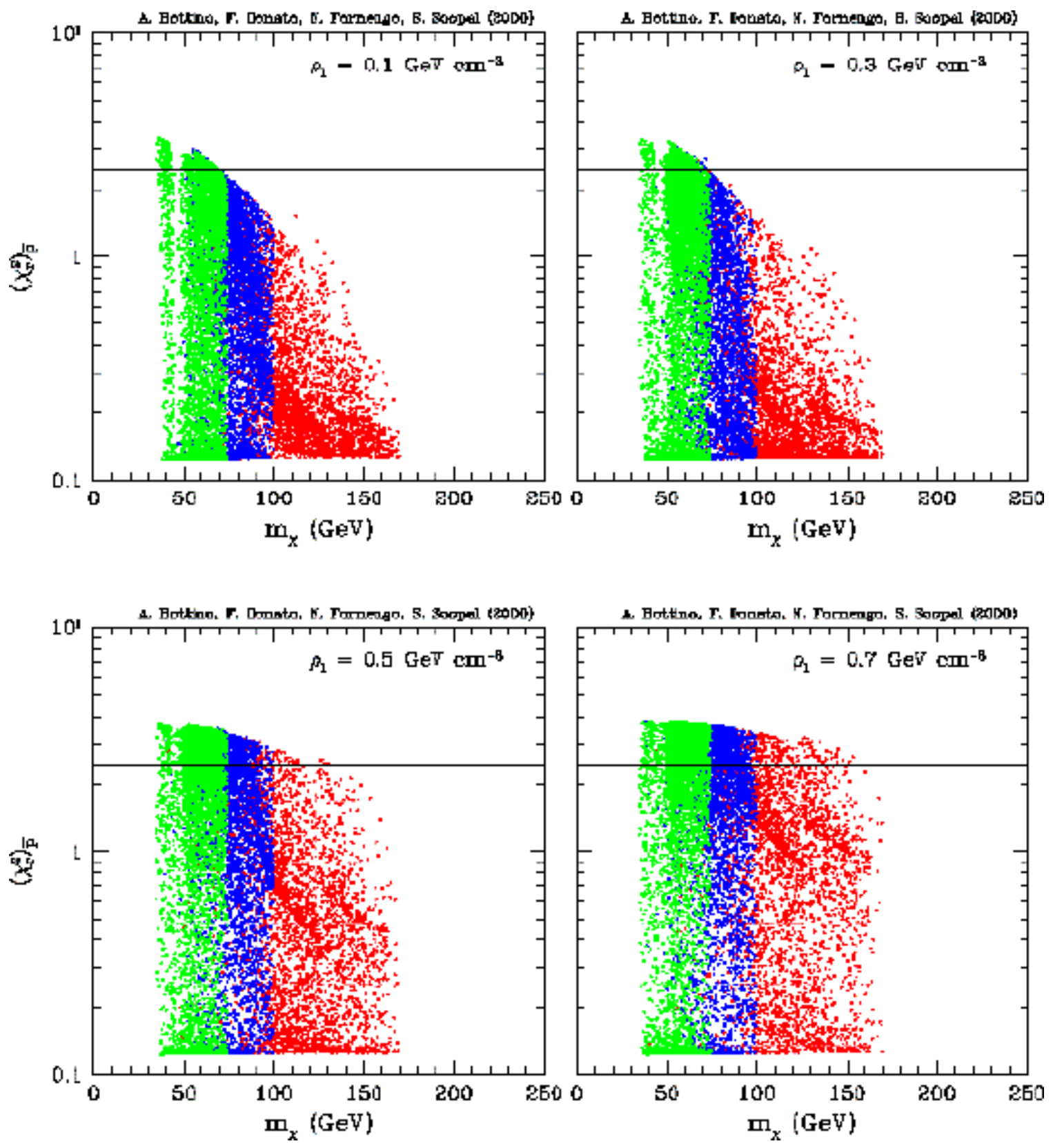

FIG. 7. Scatter plot for the reduced $\chi_{r}^{2}$ 's in a comparison of the calculated cosmic-ray antiprotons fluxes with the combined experimental data of BESS95 and BESS97 [54. The horizontal line denotes the value $\chi_{r}^{2}=2.44$, which for 13 d.o.f. corresponds to a $99.7 \%$ C.L., above which we disallow susy configurations. The grey-level (color) code is the same as in Fig. 2. 

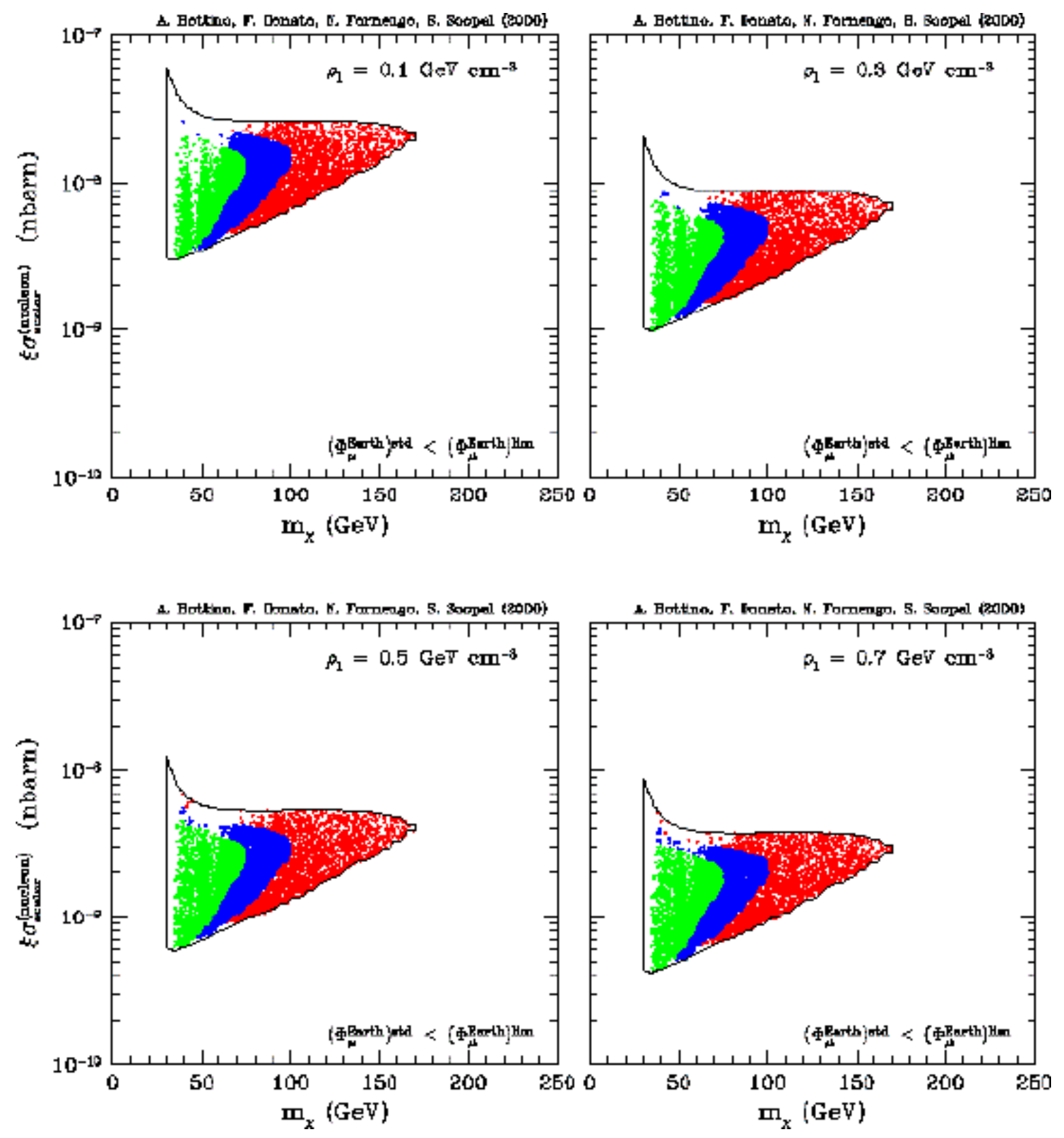

FIG. 8. As in Fig. 2, once the constraints from the up-going muon fluxes from the center of the Earth are applied, assuming a Maxwellian halo distribution for relic neutralinos. The grey-level (color) code is the same as in Fig. 2. 

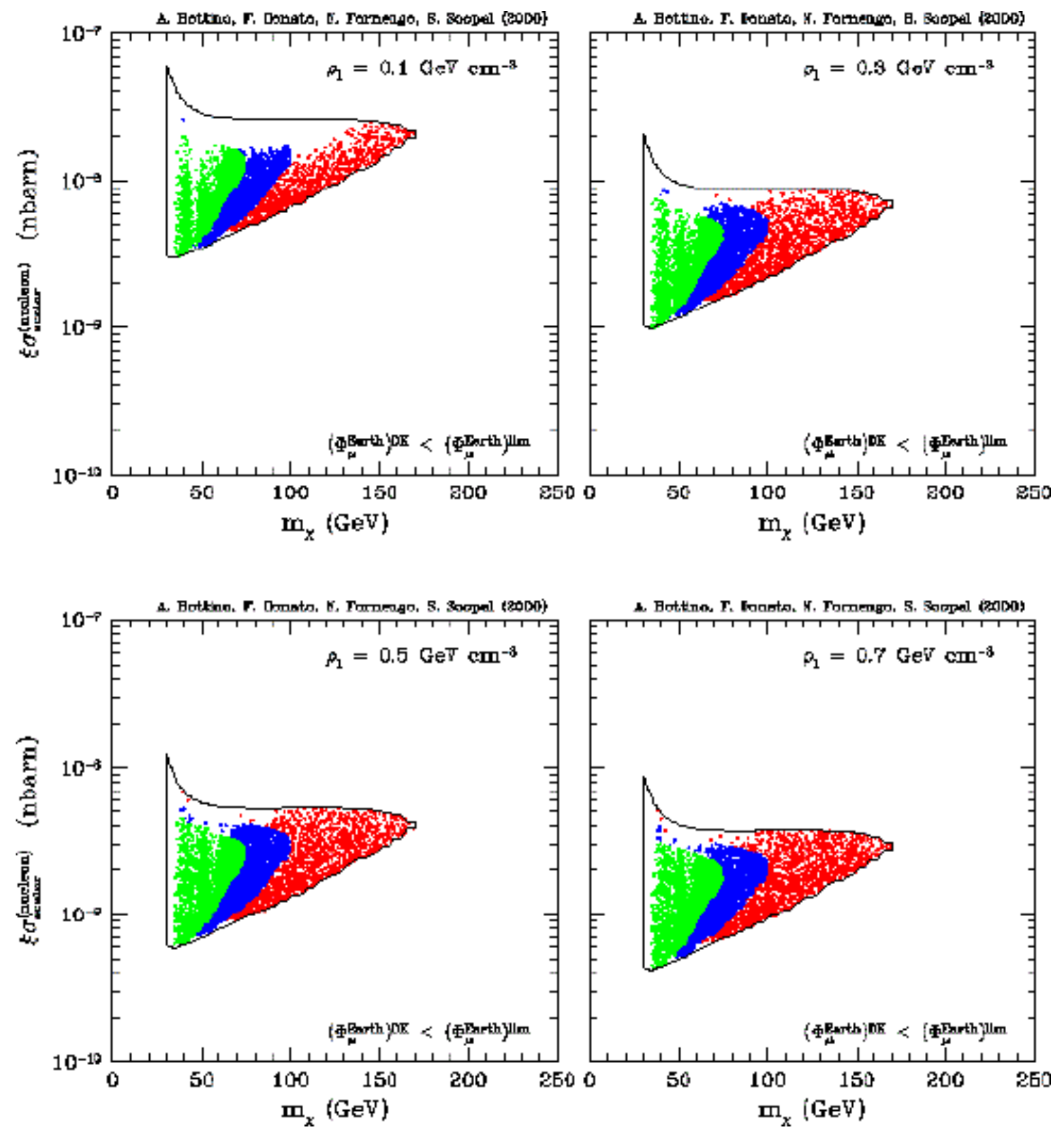

FIG. 9. Covering of the annual-modulation regions, if the constraint $\left(\Phi_{\mu}^{\text {Earth }}\right)^{\mathrm{DK}} \leq$ $\left(\Phi_{\mu}^{\text {Earth }}\right)^{\lim }$ were applied. The grey-level (color) code is the same as in Fig. 2. 

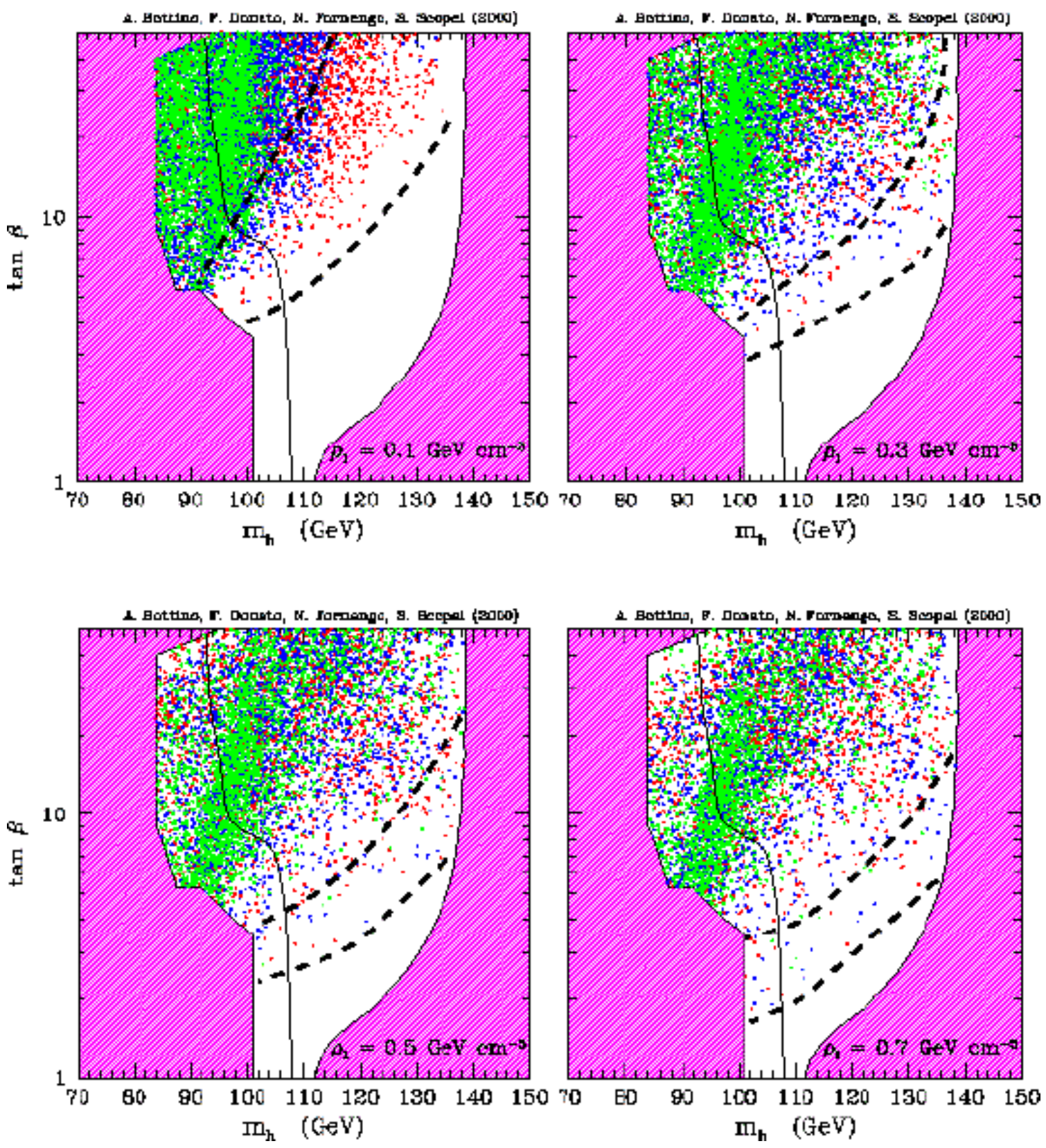

FIG. 10. Scatter plot for set $T$ in the plane $m_{h}-\tan \beta$. The grey-level (color) code is the same as in Fig. 2. For each panel, the lower dashed line denotes the frontier of the complete scatter plot; the upper dashed line denotes the frontier, when only set 1 for the hadronic quantities of Sect. II is employed. The hatched region on the right is excluded by theory. The hatched region on the left is excluded by present data from LEP [16 and CDF [17]. The solid line represents the $95 \%$ C.L. bound reachable at LEP2, in case of non discovery of a neutral Higgs boson. 

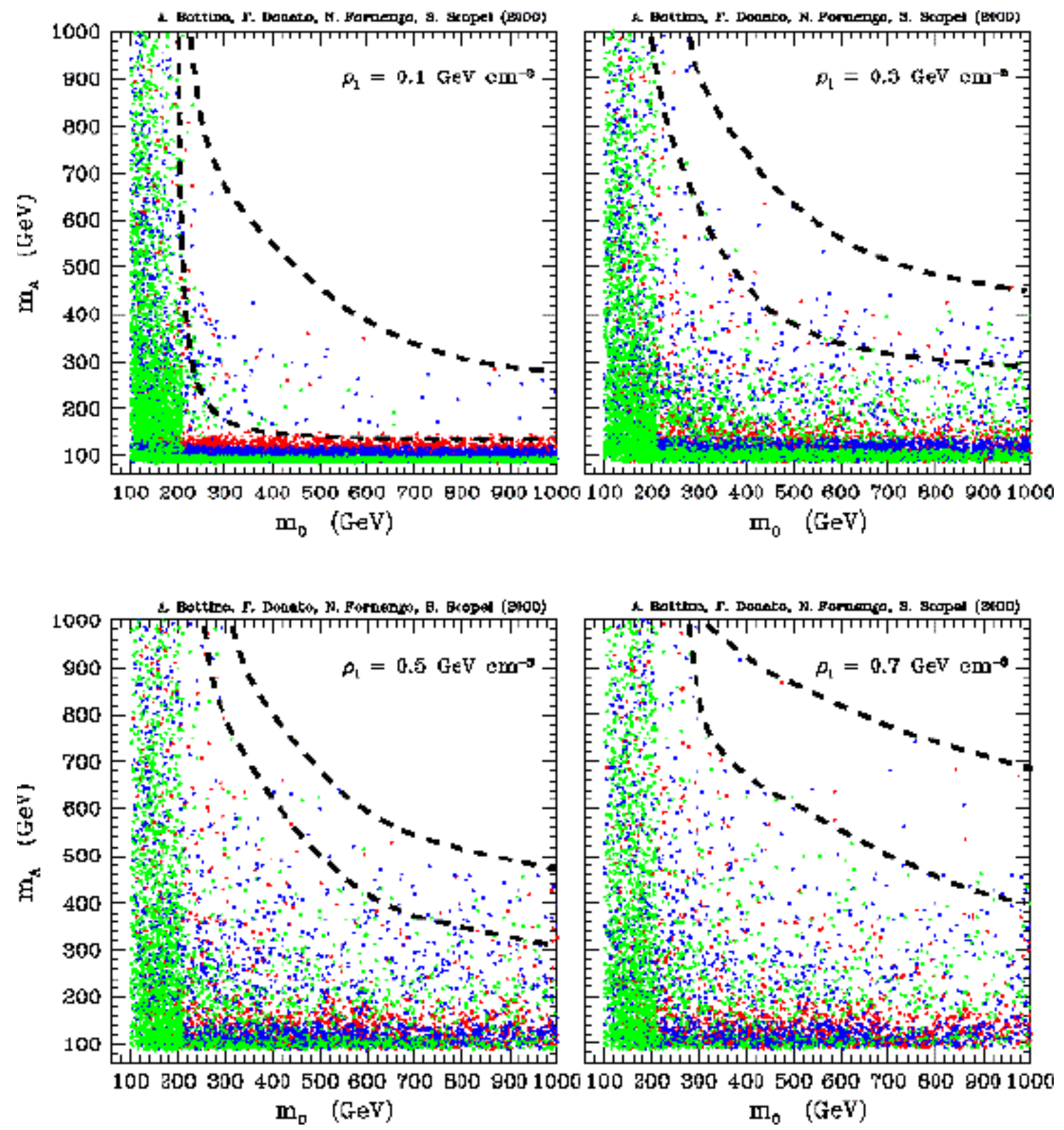

FIG. 11. Scatter plot for set $T$ in the plane $m_{0}-m_{A}$. The grey-level (color) code is the same as in Fig. 2. For each panel, the upper dashed line denotes the frontier of the complete scatter plot; the lower dashed line denotes the frontier, when only set 1 for the hadronic quantities of Sect. II is employed. 

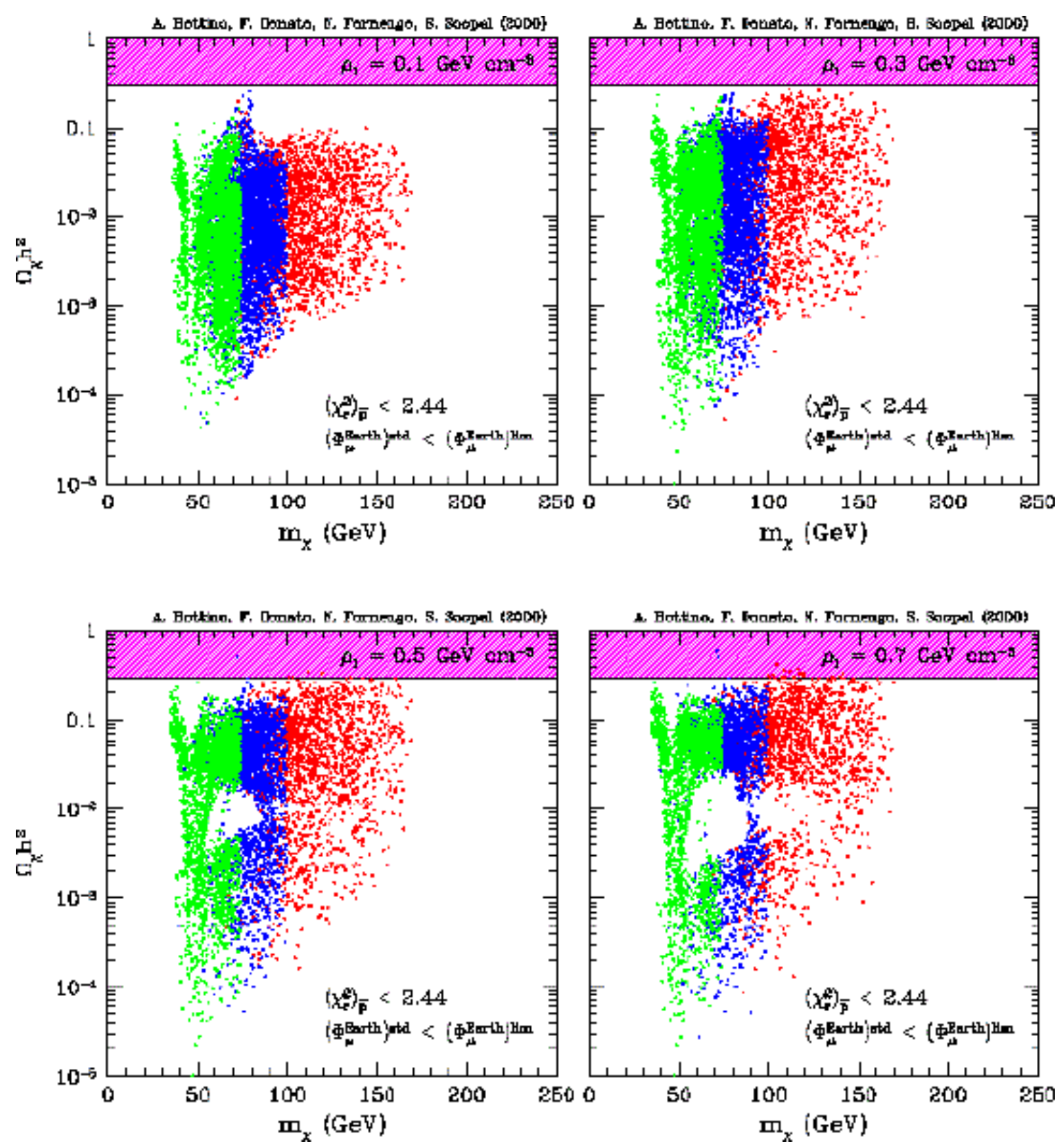

FIG. 12. Neutralino relic abundance $\Omega_{\chi} h^{2}$ versus $m_{\chi}$, once the constraints from up-going muon fluxes and cosmic-ray antiprotons are applied. The hatched region is disallowed by the upper limit on cold dark matter $\Omega_{C D M} h^{2} \lesssim 0.3[22]$. 